\title{
CATÁLOGO DE LAS ARAÑAS (ARANEAE) DE LA COMUNIDAD DE MADRID
}

\author{
M. A. Ferrández*, E. Morano*, H. Fernández de Céspedes* y M. Camargo*
}

\section{RESUMEN}

Con la confección del presente catálogo se pretende recopilar la información faunística disponible sobre las arañas de la Comunidad de Madrid, valorar el grado de conocimiento en el contexto de la fauna ibérica, así como impulsar los estudios encaminados a su conservación.

Para su elaboración se han recopilado las citas publicadas, las citas inéditas de diferentes Tesis Doctorales y de licenciatura, el material identificado que se conserva en las colecciones del Museo Nacional de Ciencias Naturales y los resultados de un muestreo puntual realizado por dos de los autores (M.A. Ferrández y H. Fernández de Céspedes) en las proximidades de San Agustín de Guadalix.

La nomenclatura está basada tanto en el catálogo mundial de Platnick (2005) como en el realizado para la fauna ibero-balear por uno de los autores, Morano (2005), disponible actualmente en internet.

Para cada especie se incluyen las localidades de captura, su coordenada UTM, el nombre con el que fue publicada la cita y la referencia bibliográfica o el museo donde esta depositado el material. También se añade un apartado de distribución donde se proporciona la distribución mundial y la ibérica.

La fauna de arañas de Madrid cuenta en el momento actual con un total de 261 especies repartidas en 132 géneros de 36 familias distintas, de las cuales un total de 52 se publican por primera vez (las señaladas mediante un *). La conclusión general es que se poseen pocos datos de la mayoría de las especies, los datos que hay son en muchos casos antiguos y no aportan información relevante sobre los ciclos biológicos, ecología, etc. Sin duda quedan muchas especies por encontrar en la fauna madrileña y no es descabellado estimar entre 350 y 400 las especies totales de dicha fauna.

También es de destacar la presencia de 24 endemismos ibéricos, algunos de ellos recolectados en fecha reciente, como Ozyptila umbraculorum (2002), Clubiona aducta, Parachtes teruelis, o Amphiledorus balnearicus (2005), esta última capturada en los muestreos de San Agustín de Guadalix.

Palabras clave: arañas, biodiversidad, Madrid, España.

\section{ABSTRACT \\ Catalogue of the spiders (Araneae) of Comunidad de Madrid}

In this catalogue we seek to compile information about spiders in Comunidad de Madrid in order to evaluate the level of our knowledge in the context of the Iberian fauna as well as to promote studies leading to their conservation.

\footnotetext{
* Sociedad para el estudio y la conservación de las arañas. C/ Villafranca n ${ }^{\circ}$ 24, $1^{\circ}$ C. 28028 Madrid.
}

e-mail: harpactea@yahoo.es 
For this catalogue we have compiled information coming from both published and unpublished mentions from university thesis, classified specimens from the collections of the Museo Nacional de Ciencias Naturales and the results of a specific sampling carried out by M. A. Ferrández and H. Fernández de Céspedes in the San Agustín de Guadalix area.

The nomenclature is based on Platnick's world catalogue (2005) as well as on Morano's work on the ibero-balearic fauna (2005) both available on the net.

For each species we include: locality of record, UTM coordinates, original publication name and bibliographical references or museum where the material is kept.

Madrid's fauna of spiders has 261 species at present, belonging to 133 genera from 36 different families, 52\% of which are cited from first time (marked with $*$ ). The general conclusion is that there is very little data about most species, those that do exist are often outdated and they lack relevant information about biological cycles, ecology, etc.

There are still great many species to be recorded in the fauna of Madrid and estimates range between 350 and 400 species in total.

It must be pointed that 24 out of 261 total species are endemic species from the Iberian Peninsula, some only collected very recently: Ozyptila umbraculorum (2002), Clubiona aducta, Parachtes teruelis (2005) and Amphiledorus balnearicus (in this paper).

Key words: spiders, biodiversity, Madrid, Spain.

\section{Introducción}

En el presente catálogo analizamos los datos disponibles sobre las arañas de la Comunidad de Madrid (CM), con el objetivo de valorar su grado de conocimiento e impulsar su estudio. Catálogos dedicados a la fauna española son los publicados por Fernández Galiano (1910), Pérez de San Román (1947) y más recientemente el de Morano (2005), disponible únicamente a través de internet (una versión anterior Morano, 2003 no está actualmente disponible). Respecto a la fauna portuguesa disponemos de las publicaciones de Bacelar (1927-1940) y la Check-list actualizada de Cardoso (2000). Para el conjunto de las montañas pirenaicas disponemos de un catálogo realizado por Bosmans \& de Keer (1985) y por último recientemente han visto la luz catálogos de comunidades autónomas: Melic (2000) para Aragón y Castro (2005) para el País Vasco, o para provincias: Sánchez (2003), para Cádiz.

Para la elaboración del catálogo hemos utilizado: 1) los datos bibliográficos, 2) las citas inéditas procedentes de tesis doctorales (Ferrández, 1987) y tesis de licenciatura (Camargo, 1982; Morano, 1984 y Fernández de Céspedes, 1987), 3) las citas inéditas procedentes del material depositado en las colecciones del Museo Nacional de Ciencias Naturales (MNCN) y 4) los resultados de los muestreos realizados en 2005 en las proximidades de San Agustín de Guadalix. Estos últimos están señalados como Ferrández y Fernández de Céspedes, sin fecha detrás.
El catálogo esta estructurado en tres apartados: Nomenclatura, citas de la Comunidad de Madrid (CM) y distribución incluyendo para cada especie 1) el nombre valido, seguido del autor, año y referencia de su publicación, así como el nombre original con el fue descrito; 2) todas las citas de la Comunidad de Madrid por orden alfabético de localidad, indicando a) el nombre científico con el fue citado, b) la coordenada UTM de $10 \mathrm{~km}$ de lado, c) la referencia bibliográfica en la que se citó originalmente (no hemos creído oportuno incluir las citas que para una misma muestra -los mismos ejemplares, de la misma localidad y fecha-, se encuentran recogidas en diferentes publicaciones, ya que consideramos que no añaden más información faunística al catálogo), d) si el material se encuentra depositado en las colecciones del Museo Nacional de Ciencias Naturales (MNCN), lo hacemos constar e indicamos tanto el numero de colección, como el responsable de su identificación, y e) en los casos en que el material citado en una publicación haya sido revisado, cambiando o no su identificación, lo hacemos constar; y 3 ) incluimos información orientativa sobre su distribución mundial e ibérica, en el primer caso hemos utilizado el catálogo de Platnick (2005), y en el segundo el de Morano (2005b).

Las familias, géneros y especies están todas ordenadas de forma alfabética y hemos seguido la nomenclatura utilizada por Platnick (2005) y las primeras citas para la comunidad de Madrid -que suman un total de 15 géneros y 52 especies- se señalan con un asterisco. 
En el catálogo se incluyen datos referentes a un total de 261 especies, pertenecientes a 133 géneros de 36 familias distintas. Esta cifra representa aproximadamente el 19\% del total de la fauna Ibérica. Este porcentaje es bajo comparado con otros grupos de artrópodos, así los Carábidos de Madrid (Ortuño, 1996) representan más del 30\% de la fauna Ibérica. No es aventurado suponer que ocurra otro tanto con las arañas, así podríamos estimar que la fauna de arañas de Madrid puede estar compuesta por 350 especies o más.

Estos valores son importantes si los comparamos, a nivel ibérico, con otros grupos más populares: aves (368 especies) (Ramos, 2002), dentro de los Insectos coleópteros (más de 10.000 especies), Dípteros (10.000-12.000 especies), Himenópteros (9.400 especies), Lepidópteros (4.223 especies), de los cuales son Ropalóceros 224 especies y 1.400 Heterópteros (Ortuño, 2002), o con otras regiones: Finlandia (536 especies), Gran Bretaña (645 especies), grupos y países que cuentan con mayor numero de investigadores.

En su conjunto los datos disponibles sobre las arañas de la Comunidad de Madrid son fraccionarios, tanto en el aspecto taxonómico, como en el geográfico y en muchos casos las citas son muy antiguas. Aproximadamente la mitad de las especies de arañas de la Comunidad de Madrid cuentan con una sola cita. En la Tabla 1 podemos apreciar que la mayoría de las familias de arañas de la Comunidad de Madrid están poco o nada estudiadas, prácticamente no existen datos sobre las familias más diversificadas a nivel mundial: Linyphiidae, Gnaphosidae, Theridiidae, y tan solo sobre unas pocas: Araneidae, Thomisidae y Dysderidae tenemos un grado aceptable de información. Las especies más citadas (ver Tabla 2) corresponden a especies comunes de amplia distribución, particularmente de las familias en las cuales se han realizado trabajos específicos Araneidae y Dysderidae (ver bibliografía). Esto es válido incluso para las especies más comunes. Por lo que respecta a las zonas estudiadas existe una gran parte del territorio de la Comunidad de Madrid sin prospectar y únicamente se han muestreado de forma intensiva y con métodos de muestreo indirecto dos localidades: Valdelatas, en los alrededores de la Universidad Autónoma de Madrid, y Valdelaguna. En ambos casos se muestreo únicamente con trampas de suelo y en el primero de los estudios no se identificaron las familias más diversificadas.

Dentro de este panorama tampoco nos debe sorprender que incluso los diferentes Espacios Naturales Protegidos no estén estén suficientemente muestrea-
Tabla 1.- Importancia relativa y nivel de conocimiento de las distintas familias de arañas de la Comunidad de Madrid.

Table 1.- Relative importance and level of knowledge of the different spider families of Comunidad de Madrid.

\begin{tabular}{|c|c|c|c|c|}
\hline Familia & $\mathrm{n}^{0}$ géneros & $n^{0}$ especies & $n^{0}$ endemismos & $n^{0}$ de citas \\
\hline Agelenidae & 4 & 9 & 2 & 45 \\
\hline Anyphaenidae & 1 & 3 & 0 & 7 \\
\hline Araneidae & 15 & 28 & 0 & 232 \\
\hline Atypidae & 1 & 1 & 0 & 2 \\
\hline Clubionidae & 1 & 1 & 1 & 3 \\
\hline Corinnidae & 1 & 1 & 1 & 3 \\
\hline Dictynidae & 1 & 1 & 0 & 2 \\
\hline Dysderidae & 4 & 8 & 4 & 77 \\
\hline Eresidae & 2 & 3 & 1 & 10 \\
\hline Filistatidae & 2 & 2 & 0 & 8 \\
\hline Gnaphosidae & 12 & 27 & 0 & 51 \\
\hline Hersiliidae & 1 & 1 & 0 & 2 \\
\hline Linyphiidae & 11 & 11 & 1 & 18 \\
\hline Liocranidae & 3 & 5 & 1 & 11 \\
\hline Lycosidae & 7 & 21 & 2 & 55 \\
\hline Mimetidae & 2 & 3 & 0 & 10 \\
\hline Miturgidae & 1 & 5 & 0 & 6 \\
\hline Nemesidae & 1 & 4 & 3 & 4 \\
\hline Nesticidae & 1 & 1 & 0 & 1 \\
\hline Oecobiidae & 2 & 3 & 0 & 6 \\
\hline Oonopidae & 1 & 2 & 1 & 2 \\
\hline Oxyopidae & 1 & 4 & 0 & 20 \\
\hline Palpimanidae & 1 & 1 & 0 & 3 \\
\hline Philodromidae & 4 & 15 & 0 & 30 \\
\hline Pholcidae & 3 & 5 & 1 & 11 \\
\hline Pisauridae & 1 & 1 & 0 & 7 \\
\hline Salticidae & 16 & 24 & 0 & 45 \\
\hline Segestriidae & 1 & 2 & 0 & 3 \\
\hline Scytodidae & 1 & 3 & 0 & 6 \\
\hline Sicariidae & 1 & 1 & 0 & 3 \\
\hline Sparassidae & 3 & 4 & 0 & 20 \\
\hline Tetragnathidae & 4 & 6 & 1 & 25 \\
\hline Therididae & 6 & 11 & 0 & 19 \\
\hline Thomisidae & 10 & 29 & 1 & 86 \\
\hline Titanoecidae & 2 & 3 & 0 & 3 \\
\hline Uloboriidae & 2 & 2 & 0 & 3 \\
\hline Zodariidae & 3 & 8 & 3 & 21 \\
\hline Total & 131 & 261 & 25 & 857 \\
\hline
\end{tabular}

do: Parque Natural de Peñalara (4), Montejo (4) Parque regional del Sureste (24), Cuenca alta del Manzanares (64), Mar de Ontígola (27) y Monte de Abantos (52).

A pesar de lo anteriormente expuesto el desconocimiento de la fauna de arañas de la Comunidad de Madrid es menor que en la mayoría de las demás comunidades autónomas peninsulares (ver Tabla 3) y por provincias, tan solo Huesca (435) y Barcelona (321) superan el numero de especies conocidas de la provincia de Madrid (255), estando a su mismo nivel la de Salamanca (235). Las demás provincias 
Tabla 2.- Las especies de arañas más citadas en la Comunidad de Madrid (con 10 citas o más).

Table 2.- Spider species with greatest number of records in Comunidad de Madrid (10 records or more).

\begin{tabular}{llc}
\hline Familia & Especie & $\mathbf{n}^{\mathbf{0}}$ de citas \\
\hline Dysderidae & Dysdera crocata & 31 \\
Araneidae & Larinoides sclopetarius & 27 \\
Araneidae & Araniella cucurbitina & 27 \\
Araneidae & Mangora acalypha & 22 \\
Dysderidae & Harpactea fageli & 20 \\
Araneidae & Agalanatea redii & 17 \\
Araneidae & Neoscona adianta & 16 \\
Araneidae & Aculepeira armida & 15 \\
Agelenidae & Tegenaria atrica & 14 \\
Sparassidae & Micrommata ligurina & 14 \\
Tetragnathidae & Tetragnatha extensa & 14 \\
Araneidae & Argiope bruennichi & 11 \\
Thomisidae & Synaema globosum & 11 \\
Dysderidae & Harpactocrates gurdus & 10 \\
Thomisidae & Thomisus onustus & 10 \\
\hline
\end{tabular}

no llegan a las 200 especies (Morano, 2005). Como botón de muestra podemos indicar que en un solo muestreo, de una sola localidad, de un solo día, de una sola persona, se pueden capturar -sin ninguna dificultad- más de 40 especies.

Como resultado de la elaboración del presente catálogo resulta evidente la necesidad de impulsar el estudio de las arañas en la Comunidad de Madrid, tanto desde del punto de vista de la investigación básica, como en los distintos aspectos relacionados con su conservación. Bajo el primer aspecto convendría impulsar los estudios sobre la distribución, biología y ecología de las distintas especies, dedicando mayor esfuerzo de muestreo a aquellas familias peor conocidas. Dentro del segundo aspecto convendría realizar inventarios de los Espacios Naturales Protegidos y valorar las posibles especies amenazadas, y promocionar su estudio.

\section{Familia Agelenidae C.L. Koch, 1837}

Agelena labyrinthica (Clerck, 1758)

Araneus labyrinthicus

CITAS DE LA CM

Agelena labyrinthica (Clerck, 1758), Pelayos, 30TUK97, Fernández Galiano, 1910

Agelena labyrinthica (Clerck, 1758), Robledo de Chavela, 30TUK97, Fernández Galiano, 1910

Agelena labyrinthica (Clerck, 1758), San Agustín de Guadalix, 30TVL40, Ferrández y Fernández de Céspedes
Tabla 3.- Biodiversidad de Arañas de España penínsular, por Comunidades Autónomas, tomado de Morano (2005a). Los datos de Madrid, son los del presente catálogo y los del País Vasco están tomados de Castro (2005).

Tabla 3.- Biodiversity of the spider fauna from different Comunidades Autónomas (regions) of Spain. Data from Morano, 2005 (Spain); Castro, 2005 (Basque Country) and this paper (Madrid).

\begin{tabular}{lcc}
\hline Comunidad Autónoma & $\mathbf{n}^{\mathbf{0}} \mathbf{s p}$ & Superficie $\mathbf{( k m}^{\mathbf{2}} \mathbf{~}$ \\
\hline Aragon & 504 & 47650 \\
Cataluña & 477 & 31895 \\
Andalucia & 426 & 87267 \\
Castilla León & 389 & 94193 \\
Madrid & $\mathbf{2 5 5}$ & $\mathbf{7 9 9 5}$ \\
Baleares & 215 & 5014 \\
Galicia & 191 & 29434 \\
Navarra & 162 & 10421 \\
Asturias & 145 & 10565 \\
Pais Vasco & 140 & 7261 \\
Extremadura & 114 & 41602 \\
Cominidad Valenciana & 114 & 23305 \\
Murcia & 78 & 11317 \\
La Rioja & 65 & 5034 \\
Cantabria & 53 & 5289 \\
\hline
\end{tabular}

Agelena labyrinthica (Clerck, 1758), San Martín de Valdeiglesias, 30TUK85, Fernández Galiano, 1910

Agelena labyrinthica (Clerck, 1758), Valdecerete, 30TVK85, Fernández Galiano, 1910

DistriBución: Paleártica. Toda la Península Ibérica.

Lycosoides coarctata (Dufour, 1831)

- Aranea coarctata

CITAS DE LA CM

Lycosoides coarctata (Dufour, 1831), Madrid (capital), 30TVK47, Ferrández \& Beamonte, 2005

Lycosoides coarctata (Dufour, 1831), Torrelodones, 30TVK29, Ferrández \& Beamonte, 2005

Textrix coarctata (Dufour, 1831), Valdelatas, 30TVK49, Camargo, 1982

DistRIBUCión: Mediterránea, incluyendo las islas atlánticas. Casi toda la península, falta en Galicia y cornisa cantábrica.

Tegenaria atrica C.L. Koch, 1843

- Tegenaria atrica

CitAs DE LA CM

Tegenaria atrica C.L. Koch, 1843, Aldea de Fresno, 30TUK96, Barrientos \& Ribera, 1988

Tegenaria atrica C.L. Koch, 1843, Cercedilla, 30TVL11, Barrientos \& Ribera, 1988

Tegenaria atrica C.L. Koch, 1843, Cercedilla. Cerro Colgado, 30TVL11, Barrientos \& Ribera, 1988

Tegenaria atrica C.L. Koch, 1843, Cercedilla. Estación Alpina, 30TVL11, Barrientos \& Ribera, 1988 
Tegenaria nervosa Simon, 1870, El Escorial, 30TVK09, Fernández Galiano, 1910

Tegenaria atrica C.L. Koch, 1843, La Acebeda, 30TVL44, Ferrández \& Beamonte, 2005

Tegenaria atrica C.L. Koch, 1843, Madrid, 30TVK47, Barrientos \& Ribera, 1988

Tegenaria atrica C.L. Koch, 1843, Pinto, 30TVK57, Sanz de Diego, 1885

Tegenaria atrica C.L. Koch, 1843, Pozuelo, 30TVK37, Barrientos \& Ribera, 1988

Tegenaria atrica C.L. Koch, 1843, Pto de Malagón, 30TVK09, Barrientos \& Ribera, 1988

Tegenaria nervosa Simon, 1870, Robledo de Chavela, 30TUK97, Fernández Galiano, 1910

Tegenaria atrica C.L. Koch, 1843, Torrelodones, 30TVK29, Ferrández \& Beamonte, 2005

Tegenaria atrica C.L. Koch, 1843, San Agustín de Guadalix, 30TVL40, Ferrández y Fernández de Céspedes

Tegenaria atrica C.L. Koch, 1843, Valdelatas, 30TVK49, Camargo, 1982

DisTRIBUCIÓN: Europea. Repartida por toda la Península.

Tegenaria bucculenta (L. Koch, 1868)

- Coelotes bucculentus

CiTAS DE LA CM

Tegenaria bucculenta Koch, 1868, La Acebeda, 30TVL44 Ferrández y Beamonte, 2005

Coelotes bucculentus L. Koch, 1868, El Escorial, 30TVK09, L. Koch, 1868

Tegenaria bucculenta L. Koch, 1868, Pelayos, 30TUK97, Fernández Galiano, 1910

Tegenaria bucculenta L. Koch, 1868, Pto del Paular, 30TVL22, Brignoli, 1978

Tegenaria bucculenta C.L. Koch, 1868, Robledo de Chavela, 30TUK97, Fernández Galiano, 1910

DisTRIBUCIÓN: Endemismo Ibérico. Presente en la mitad sur de la Península Ibérica.

Tegenaria domestica (Clerck, 1758)

- Araneus domesticus

CitAs DE LA CM

Tegenaria domestica (Clerck, 1758), Madrid, 30TVK47, Fernández Galiano, 1910

DistriBuCiÓn: Cosmopolita. Toda la Península.

Tegenaria feminea Simon, 1870

- Tegenaria feminea

CitAs DE LA CM

Tegenaria cisticola Simon, 1870, Aranjuez, 30TVK53, Sanz de Diego, 1885

Tegenaria carpetana Brignoli, 1978, Galapagar, 30TVK19, Brignoli, 1979

Tegenaria cisticola Simon, 1870, Madrid, 30TVK47, Simon, 1875

Tegenaria feminea Simon, 1870, San Agustín de Guadalix, 30TVL40, Ferrández y Fernández de Céspedes

Tegenaria feminea Simon, 1870, Valdelaguna, 30TVK64, Fernández de Céspedes, 1987

Tegenaria feminea Simon, 1870, Valdelatas, 30TVK49, Camargo, 1982

Distribución. Endemismo Ibérico. Toda la Península.
Tegenaria montigena Simon, 1937

- Tegenaria montigena

CitAs DE LA CM

Tegenaria montigena Simon, 1937, La Acebeda, 30TVL44, Ferrández \& Beamonte, 2005

Tegenaria montigena Simon, 1937, Peñalara, 30TVL12, Simon, 1937

DisTRIBUCIÓN. Mediterránea occidental. Solo en la mitad Norte de la Península.

Tegenaria picta Simon, 1870

- Tegenaria picta

CitAs DE LA CM

Tegenaria minuta Simon, 1870, Sierra de Guadarrama, Simon, 1870

Tegenaria picta Simon, 1870, Sierra de Guadarrama, Simon, 1875

Tegenaria picta Simon, 1870, Valdelaguna, 30TVK64, Fernández de Céspedes, 1987

Tegenaria picta Simon, 1870, Valdelatas, 30TVK49, Camargo, 1982

Distribución. Europa y norte de África. Toda la Península Ibérica.

\section{Tegenaria sp.}

CitAs DE LA CM

Tegenaria sp, Patones. Cv del Reguerillo, 30TVL51, Machado, 1942, material depositado en el MNCN 20.02/10503

Textrix caudata L. Koch, 1872

- Textrix caudata

CITAS DE LA CM

Textrix caudata L. Koch, 1872, El Escorial, 30TVK09, Schenckel, 1938

Textrix caudata L. Koch, 1872, San Agustín de Guadalix, 30TVL40, Ferrández y Fernández de Céspedes

Textrix caudata L. Koch, 1872, Valdelatas, 30TVK49, Camargo, 1982

Distribución. Mediterránea, incluyendo las islas Atlánticas.

\section{Textrix sp}

CitAS DE LA CM

Textrix sp, Madrid (Capital), 30TVK47, Fernández Bourgon, 2003

Familia Anyphaenidae Bertkau, 1878

Anyphaena accentuata (Walckenaer, 1802)

- Aranea accentuata

CitAs DE LA CM

Anyphaena accentuata (Walckenaer, 1802), Aldea del Fresno, 30TUK97, Urones, 1996

Distribución. Europa y Asia central. Mitad norte de la Península Ibérica. 
Anyphaena alboirrorata Simon, 1878

- Anyphaena alboirrorata

CITAS DE LA CM

Anyphaena alboirrorata Simon, 1878, Madrid. Casa de Campo, 30TVK47, Urones, 1996

DisTRIBUCión. Mediterránea occidental. Mitad norte de la Península Ibérica.

Anyphaena numida Simon, 1897

- Anyphaena numida

CiTAS DE LA CM

Anyphaena numida Simon, 1897, Aldea del Fresno, 30TUK97, Urones, 1996

Anyphaena numida Simon, 1897, Aranjuez, 30TVK53, Urones, 1996

Anyphaena numida Simon, 1897, El Escorial, 30TVK09, Urones, 1996

Anyphaena numida Simon, 1897, Madrid, 30TVK47, Urones, 1996

Anyphaena numida Simon, 1897, Torrelodones, 30TVK29, Ferrández \& Beamonte, 2005

DistRIBUCIÓN. Mediterráneo occidental. Mitad norte de la Península Ibérica.

Familia Araneidae Latreille, 1806

Aculepeira armida (Savigny \& Audouin, 1826)

- Epeira armidara

CITAS DE LA CM

Epeira armida (Savigny \& Audouin, 1826), Aranjuez, 30TVK53, Sanz de Diego, 1885

Aculepeira armida (Savigny \& Audouin, 1826), VelillaArganda, 30TVK56, Morano \& Ferrández, 1986

Araneus armida (Savigny \& Audouin, 1826), El Escorial, 30TVK09, Fernández Galiano, 1910. Depositado en el MNCN 20.02/10438

Aculepeira armida (Savigny \& Audouin, 1826), El Berrueco, 30TVL52, Jiménez, 2002

Aculepeira armida (Savigny \& Audouin, 1826), Hoyo de Manzanares, 30TVK29, Morano \& Ferrández, 1986

Araneus armida (Savigny \& Audouin, 1826), Madrid, 30TVK47, Fernández Galiano, 1910

Epeira armida (Savigny \& Audouin, 1826), Madrid (capital), 30TVK47, Sanz de Diego, 1885

Aculepeira armida (Savigny \& Audouin, 1826), Madrid-Burgos km 70, 30TVL43, Morano \& Ferrández, 1986

Aculepeira armida (Savigny \& Audouin, 1826), Manzanares el Real, 30TVL31, Morano \& Ferrández, 1986

Aculepeira armida (Savigny \& Audouin, 1826), Navas del Rey, 30TVL47, Morano \& Ferrández, 1986

Aculepeira armida (Savigny \& Audouin, 1826), Robledo de Chavela, 30TUK97, Morano \& Ferrández, 1986

Aculepeira armida (Savigny \& Audouin, 1826), Robledo de Chavela, 30TUK97, Fernández Galiano, 1910. Depositado en el MNCN 20.02/10423

Aculepeira armida (Savigny \& Audouin, 1826), Soto del Real, 30TVL31, Morano \& Ferrández, 1986
Aculepeira armida (Savigny \& Audouin, 1826), Valdevivar, 30TVK54, Morano \& Ferrández, 1986

Aculepeira armida (Savigny \& Audouin, 1826), Viñuelas, 30TVK49, Morano \& Ferrández, 1986

Distribución. Paleártica occidental. Toda la Península.

Aculepeira ceropegia (Walckenaer, 1802)

- Aranea ceropegia

Citas DE LA CM

Araneus ceropegius (Walckenaer, 1802), El Escorial, 30TVK09, Fernández Galiano, 1910

Aculepeira ceropegia (Walckenaer, 1802), Pto de la Morcuera, 30TVL31, Morano \& Ferrández, 1986

Aculepeira ceropegia (Walckenaer, 1802), Pto del Reventon, Arryo de Calderuelos, 30TVL32, Morano \& Ferrández, 1986 Aculepeira ceropegia (Walckenaer, 1802), Robledo de Chavela, 30TUK97, Morano \& Ferrández, 1986

Araneus ceropegia (Walckenaer, 1802), Robledo de Chavela, 30TUK97, Fernández Galiano, 1910

Aculepeira ceropegia (Walckenaer, 1802), Miraflores-Canencia $\mathrm{km} \mathrm{4,} \mathrm{30TVL32,} \mathrm{Morano} \mathrm{\&} \mathrm{Ferrández,} 1986$

Aculepeira ceropegia (Walckenaer, 1802), Navacerrada, 30TVL11, Morano \& Ferrández, 1986

DistrIBUCIÓN. Paleártica. Toda Iberia.

Agalanatea redii (Scopoli, 1763)

- Aranea Redii

Citas DE LA CM

Agalanatea redii (Scopoli, 1763), Alcorcón, 30TUK37, Morano \& Ferrández, 1986

Agalanatea redii (Scopoli, 1763), Aranjuez, 30TVK53, Morano \& Ferrández, 1986

Agalanatea redii (Scopoli, 1763), Velilla-Arganda, 30TVK56, Morano \& Ferrández, 1986

Agalanatea redii (Scopoli, 1763), El Berrueco, 30TVL53, Morano \& Ferrández, 1986

Agalanatea redii (Scopoli, 1763), El Berrueco, 30TVL53, Jiménez, 2002

Agalanatea redii (Scopoli, 1763), El Cuadrón, 30TVL44, Morano \& Ferrández, 1986

Araneus Redii (Scopoli, 1763), El Escorial, 30TVK09, Fernández Galiano, 1910. Depositado en el MNCN 20.02/10445

Agalanatea redii (Scopoli, 1763), El Pardo, 30TVK38, Morano \& Ferrández, 1986

Agalanatea redii (Scopoli, 1763), La Cabrera, 30TVL53, Morano \& Ferrández, 1986

Agalanatea redii (Scopoli, 1763), Madrid. Dehesa de la Villa, 30TVK48, Morano \& Ferrández, 1986

Agalanatea redii (Scopoli, 1763), Mingorrubio, 30TVK39, Morano \& Ferrández, 1986

Agalanatea redii (Scopoli, 1763), Montarco, 30TVK57, Morano \& Ferrández, 1986

Araneus Redii (Scopoli, 1763), Pelayos, 30TUK97, Fernández Galiano, 1910

Agalanatea redii (Scopoli, 1763), San Agustín de Guadalix, 30TVL40, Ferrández y Fernández de Céspedes

Araneus Redii (Scopoli, 1763), San Martín de Valdeiglesias, 30TUK85, Fernández Galiano, 1910. Depositado en el MNCN 20.02/10424

Araneus redii (Scopoli, 1763), Valdelatas, 30TVK49, Camargo, 1982 
Agalanatea redii (Scopoli, 1763), Villanueva del Pardillo, 30TVK28, Morano \& Ferrández, 1986

DisTRIBUCIÓN. Paleártica. Toda Iberia.

Araneus angulatus Clerck, 1758

- Araneus angulatus

CitAs DE LA CM

Araneus angulatus Clerck, 1758, Alpedrete, 30TVK10, Morano \& Ferrández

Epeira angulata (Clerck, 1758), El Escorial, 30TVK09, Bolívar, 1875

Araneus angulatus Clerck, 1758, Robledo de Chavela, 30TUK97, Fernández Galiano. E, 1910

Araneus angulatus Clerck, 1758, San Martín de Valdeiglesias, 30TUK85, Fernández Galiano. E, 1910. Depositado en el MNCN 20.02/10422

Araneus angulatus Clerck, 1758, Soto del Real, 30TVL31, Morano \& Ferrández, 1986

Araneus angulatus Clerck, 1758, Viñuelas, 30TVK49, Morano \& Ferrández, 1986

Distribución. Paleártica. Mitad norte de la Península Ibérica.

\section{Araneus diadematus Clerck, 1758}

- Araneus diadematus

CITAS DE LA CM

Araneus diadematus Clerck, 1758, Miraflores de la Sierra, 30TVL31, Morano \& Ferrández, 1986

Araneus diadematus Clerck, 1758, Navacerrada. Fte de los Geologos, 30TVL11, Morano \& Ferrández, 1986

Epeira diadema (Clerck, 1758), Pinto, 30TVK57, Sanz de Diego, 1885

DisTRIBUCIÓN. Holártica. Repartida por toda la Península Ibérica.

\section{Araneus pallidus (Olivier, 1789)}

- Aranea pallida

CitAs DE LA CM

Araneus pallidus (Olivier, 1789), Alcobendas, 30TVK48, Morano \& Ferrández, 1986

Araneus pallidus (Olivier, 1789), Embalse del Vellón, 30TVL41, Morano \& Ferrández, 1986

Araneus pallidus (Olivier, 1789), Leganés, 30TVK36, Morano \& Ferrández, 1986

Araneus pallidus (Olivier, 1789), Madrid. Ciudad Universitaria, 30TVK37, Morano \& Ferrández, 1986

Araneus pallidus (Olivier, 1789), Miraflores-Canencia, 30TVL33, Morano \& Ferrández, 1986

Araneus pallidus (Olivier, 1789), Soto de Viñulas, 30TVK49, Morano \& Ferrández, 1986

Araneus pallidus (Olivier, 1789), Torrelodones, 30TVK29, Morano \& Ferrández, 1986

Araneus pallidus (Olivier, 1789), Valdemaqueda, 30TUK98, Morano \& Ferrández, 1986

DisTRIBUCIÓN. Mediterráneo occidental. Toda Iberia.
Araniella alpica (L. Koch, 1869)

- Epeira alpica

CitAS DE LA CM

Araniella alpica (L. Koch, 1869), Cercedilla, 30TVL11, Morano \& Ferrández, 1986

Araniella alpica (L. Koch, 1869), Peñalara-La Granja de San Ildefonso, 30TVL12, Morano \& Ferrández, 1986

DistriBuCión. Europea. Sistema central.

\section{Araniella cucurbitina (Clerck, 1758)}

- Araneus cucurbitinus

Citas de LA CM

Araniella cucurbitina (Clerck, 1758), Alcorcón, 30TUK37, Morano \& Ferrández, 1986

Araniella cucurbitina (Clerck, 1758), Cercedilla, 30TVL11, Morano \& Ferrández, 1986

Araniella cucurbitina (Clerck, 1758), El Berrueco, 30TVL53, Morano \& Ferrández, 1986

Araniella cucurbitina (Clerck, 1758), El Cuadrón, 30TVL44, Morano \& Ferrández, 1986

Araniella cucurbitina (Clerck, 1758), El Escorial. Mte Abantos, 30TVK09, Morano \& Ferrández, 1986

Araniella cucurbitina (Clerck, 1758), El Pardo, 30TVK38, Morano \& Ferrández, 1986

Araniella cucurbitina (Clerck, 1758), Embalse de Santillana, 30TVL31, Morano \& Ferrández, 1986

Araniella cucurbitina (Clerck, 1758), Guadalix de la Sierra, 30TVL41, Morano \& Ferrández, 1986

Araniella cucurbitina (Clerck, 1758), La Cabrera, 30TVL53, Morano \& Ferrández, 1986

Araniella cucurbitina (Clerck, 1758), La Pedriza, 30TVL22, Morano \& Ferrández, 1986

Araniella cucurbitina (Clerck, 1758), Lozoya. Arryo de la Fuensanta, 30TVL33, Morano \& Ferrández, 1986

Epeira cucurbitina (Clerck, 1758), Madrid, 30TVK47, Sanz de Diego, 1885

Araniella cucurbitina (Clerck, 1758), Miraflores de la Sierra, 30TVL31, Morano \& Ferrández, 1986

Araniella cucurbitina (Clerck, 1758), Navacerrada, 30TVL11, Morano \& Ferrández, 1986

Araniella cucurbitina (Clerck, 1758), Pto de Canencia, 30TVL32, Morano \& Ferrández, 1986

Araniella cucurbitina (Clerck, 1758), Pto de Canencia. Arryo Sestil del Maillo, 30TVL32, Morano \& Ferrández, 1986

Araniella cucurbitina (Clerck, 1758), Pto del Reventón, 30TVL32, Morano \& Ferrández, 1986

Araniella cucurbitina (Clerck, 1758), Pto del Reventón. Arryo de Calderuelos, 30TVL32, Morano \& Ferrández, 1986

Araniella cucurbitina (Clerck, 1758), Pto del Reventón. Arryo Jobalo, 30TVL32, Morano \& Ferrández, 1986

Araniella cucurbitina (Clerck, 1758), Rascafría, 30TVL22, Morano \& Ferrández, 1986

Araneus cucurbitinus Clerck, 1758, Robledo de Chavela, 30TUK97, Morano \& Ferrández, 1986

Araniella cucurbitina (Clerck, 1758), Robledo de Chavela, 30TUK97, Morano \& Ferrández, 1986

Araniella cucurbitina (Clerck, 1758), San Agustín de Guadalix, 30TVL40, Ferrández y Fernández de Céspedes

Araniella cucurbitina (Clerck, 1758), Soto del Real, 30TVL31, Morano \& Ferrández, 1986

Araniella cucurbitina (Clerck, 1758), Villanueva del Pardillo, 30TVK28, Morano \& Ferrández, 1986 
Araneus cucurbitinus Clerck, 1758, Valdelatas, 30TVK49, Morano \& Ferrández, 1986

Araniella cucurbitina (Clerck, 1758), Viñuelas, 30TVK49, Morano \& Ferrández, 1986

DisTRIBUCIÓN. Paleártica. Repartida por toda la Península.

Araniella opistographa (Kulczynski, 1905)

- Araniella cucurbitina opistographa

CitAs DE LA CM

Araniella opistographa (Kulczynski, 1905), Cercedilla, 30TVL11, Jiménez-Valverde et al., 2004

Araniella opistographa (Kulczynski, 1905), Colmenar Viejo, 30TVL30, Jiménez-Valverde et al., 2004

Araniella opistographa (Kulczynski, 1905), El Berrrueco, 30TVL52, Jiménez-Valverde et al., 2004

Araniella opistographa (Kulczynski, 1905), La Acebeda, 30TVL45, Jiménez-Valverde et al., 2004

Araniella opistographa (Kulczynski, 1905), Pinilla del Valle, 30TVL32, Jiménez-Valverde et al., 2004

DistribuCIÓN. Europa hasta Asia Central. Únicamente citada de Madrid y Asturias.

Argiope bruennichi (Scopoli, 1772)

- Aranea Bruennichii

CITAS DE LA CM

Argiope bruennichi (Scopoli, 1772), Arganda-Morata de Tajuña, Km 3, 30TVK66, Morano \& Ferrández, 1986

Argiope bruennichi (Scopoli, 1772), Madrid-Valencia Km 15, 30TVK16, Morano \& Ferrández, 1986

Argiope bruennichi (Scopoli, 1772), El Pardo, 30TVK38, Morano \& Ferrández, 1986

Argiope bruennichi (Scopoli, 1772), El Escorial, 30TVK09, Bolívar, 1875

Argiope bruennichi (Scopoli, 1772), Leganes, 30TVK36, Morano \& Ferrández, 1986

Argiope bruennichi (Scopoli, 1772), Madrid (capital), 30TVK47, Fernández Galiano, 1910. Depositado en el MNCN $20.02 / 10420$

Argiope bruennichi (Scopoli, 1772), Madrid (Ciudad Universitaria), 30TVK37, Morano \& Ferrández, 1986

Argiope bruennichi (Scopoli, 1772), San Fernando de Henares, 30TVK57, Morano \& Ferrández, 1986

Argiope bruennichi (Scopoli, 1772), Titulcia, 30TVK54, Morano \& Ferrández, 1986

Argiope bruennichi (Scopoli, 1772), Torrelodones, 30TVK29, Morano \& Ferrández, 1986

Argiope bruennichi (Scopoli, 1772), Valdelatas, 30TVK49, Camargo, 1982

Distribucion. Paleártica. Toda la Península.

Argiope lobata (Pallas, 1772)

- Aranea lobata

CITAS DE LA CM

Argiope lobata (Pallas, 1772), Cenicientos, 30TUK78, Morano \& Ferrández, 1986

Argiope lobata (Pallas, 1772), Loeches, 30TVK67, Bolívar, 1875
Argiope lobata (Pallas, 1772), Madrid, 30TVK47, Dufour, 1861 Argiope lobata (Pallas, 1772), Madrid (Carabanchel), 30TUK48, Pérez Arcas, 1872

Argiope lobata (Pallas, 1772), Montarco, 30TVK57, Morano \& Ferrández, 1986

Argiope lobata (Pallas, 1772), Montarco, 30TVK57, Fernández Galiano, 1910

Argiope lobata (Pallas, 1772), San Fernando de Henares, 30TVK57, Morano \& Ferrández, 1986

Argiope lobata (Pallas, 1772), Soto de Viñuelas, 30TVK49, Morano \& Ferrández, 1986

DisTRIBUCIÓN. Mediterránea occidental. Toda la Península.

Cercidia prominens (Westring, 1851)

- Epeira prominens

Citas DE LA CM

Cercidia prominens (Westring, 1851), Pto de Morcuera, 30TVL31, Morano \& Ferrández, 1986

DistribuCiÓN. Holártica. Mitad norte de la Península.

Cyclosa algerica Simon, 1885

- Cyclosa algerina

Citas DE LA CM

Cyclosa algerica Simon, 1885, Aldea del Fresno, 30TUK97, Morano \& Ferrández, 1986

Cyclosa algerica Simon, 1885, El Berrueco, 30TVL53, Morano \& Ferrández, 1986

Cyclosa algerica Simon, 1885, El Pardo, 30TVK38, Morano \& Ferrández, 1986

Cyclosa algerica Simon, 1885, Redueñas, 30TVL25, Morano \& Ferrández, 1986

Cyclosa algerica Simon, 1885, Robledo de Chavela, 30TUK97, Morano \& Ferrández, 1986

Cyclosa algerica Simon, 1885, Villanueva del Pardillo, 30TVK28, Morano \& Ferrández, 1986

Cyclosa algerica Simon, 1885, Viñuelas, 30TVK49, Morano \& Ferrández, 1986

DisTRIBUCIÓN. Mediterráneo occidental. Toda la Península.

Cyclosa conica (Pallas, 1772)

- Aranea conica

Citas DE La CM

Cyclosa conica (Pallas, 1772), Aranjuez, 30TVK53, Sanz de Diego, 1885

Cyclosa conica (Pallas, 1772), El Berrueco, 30TVL53, Morano \& Ferrández, 1986

Cyclosa conica (Pallas, 1772), El Berrueco, 30TVL53, Jiménez, 2002

Cyclosa conica (Pallas, 1772), Hoyo de Manzanares, 30TVK29, Morano \& Ferrández, 1986

Cyclosa conica (Pallas, 1772), La Pedriza, 30TVL22, Morano \& Ferrández, 1986

Cyclosa conica (Pallas, 1772), Madrid, 30TVK47, Fernández Galiano, 1910

Cyclosa conica (Pallas, 1772), Pelayos de la Sierra, 30TUK97, Fernández Galiano, 1910. Depositado en el MNCN 20.02/ 10439

Cyclosa conica (Pallas, 1772), Pto del Reventón, 30TVL32, Morano \& Ferrández, 1986 
Cyclosa conica (Pallas, 1772), San Agustín de Guadalix, Ferrández y Fernández de Céspedes

Distribución. Holártica. Toda la Península Ibérica.

Cyclosa insulana (Costa, 1834)

- Epeira insulana

Citas de LA CM

Cyclosa oculata (Walckenaer, 1802), Montarco, 30TVK57,

Pérez de San Román, 1947 rectificación de la identificación en Morano \& Ferrández, 1986

Cyclosa insulana (Walckenaer, 1802), Pinto, 30TVK57, Sanz de Diego, 1885

DisTRIBUCIÓN. Mediterránea. Mitad norte de la Península.

\section{Cyclosa sp}

CiTAS DE LA CM

Cyclosa sp, El Molar, 30TVK57. Depositado en el MNCN $20.02 / 10437$

Cyclosa sp, El Escorial, 30TVK09. Depositado en el MNCN $20.02 / 10526$

Gibbaranea bituberculata (Walckenaer, 1802)

- Aranea bituberculata

CITAS DE LA CM

Gibbaranea bituberculata (Walckenaer, 1802), Aranjuez, 30TVK53, Morano \& Ferrández, 1986

Gibbaranea bituberculata (Walckenaer, 1802), Cantoblanco, 30TVK49, Morano \& Ferrández, 1986

Araneus dromedarius (Walckenaer, 1802), El Escorial, 30TVK09, Fernández Galiano, 1910. Depositado en el MNCN 20.02/10443

Gibbaranea bituberculata (Walckenaer, 1802), El Berrueco, 30TVL52, Jiménez, 2002

Gibbaranea bituberculata (Walckenaer, 1802), Guadalix de la Sierra. Embalse del Vellón, 30TVL41, Morano \& Ferrández, 1986

Gibbaranea bituberculata (Walckenaer, 1802), Hoyo de

Manzanares, 30TVK29, Morano \& Ferrández, 1986

Gibbaranea bituberculata (Walckenaer, 1802), Mingorrubio, 30TVK39, Morano \& Ferrández, 1986

Gibbaranea bituberculata (Walckenaer, 1802), San Agustín de Guadalix, 30TVL40, Ferrández y Fernández de Céspedes

Gibbaranea bituberculata (Walckenaer, 1802), Valdelatas, 30TVK49, Camargo, 1982

DistribuCIÓN: Paleártica occidental. Toda la Península.

Gibbaranea gibbosa (Walckenaer, 1802)

- Aranea gibbosa

CiTAS DE LA CM

Gibbaranea gibbosa (Walckenaer, 1802), Madrid!, Morano \& Ferrández, 1986

DistriBuCión: Paleártica occidental. Citada de unas pocas localidades de España y Portugal.

Hypsosinga albovittata (Westring, 1851)

- Singa albovittata

CiTAS DE LA CM

Hypsosinga albovittata (Westring, 1851), Colmenar de la SierraGuadalix km. 5, 30TVK30, Morano \& Ferrández, 1986
Hypsosinga albovittata (Westring, 1851), El Berrueco, 30TVL52, Jiménez, 2002

Hypsosinga albovittata (Westring, 1851), La Cabrera, 30TVL53, Morano \& Ferrández, 1986

Hypsosinga albovittata (Westring, 1851), Meco. Finca «El Encín», 30TVK69, Morano \& Ferrández, 1986

Hypsosinga albovittata (Westring, 1851), Viñuelas, 30TVK49, Morano \& Ferrández, 1986

Distribución: Paleártica occidental. Toda la Península.

Hypsosinga pygmaea (Sundevall, 1831)

- Theridia pygmaea

Citas de LA CM

Singa pygmaea (Sundevall, 1831), Pinto, 30TVK57, Sanz de Diego, 1885

Hypsosinga pygmaea (Sundevall, 1831), Pto del Reventón, 30TVL32, Morano \& Ferrández, 1986

DisTRIBUCIÓN: Holártica. Citada de unas pocas localidades dispersas de España y Portugal.

Hypsosinga sanguinea (C.L. Koch, 1844)

- Singa sanguinea

CITAS DE LA CM

Hypsosinga sanguinea (C.L. Koch, 1845), Bustarviejo. Arryo de Gargueña, 30TVL32, Morano \& Ferrández, 1986

Distribución: Paleártica. Mitad norte de la Península.

\section{Hypsosinga sp}

CITAS DE LA CM

Hypsosinga sp, Valdelaguna, 30TVK64, Fernández de Céspedes, 1987

Larinoides suspicax (O. Pickard-Cambridge, 1876)

- Epeira suspicax

CitAS DE LA CM

Larinoides suspicax (O. Pickard-Cambridge, 1876), Aranjuez, 30TVK53, Morano, 2002

Larionoides foliatus Sckrank, El Pardo, 30TVK38, (Kulczynski, 1901), Morano \& Ferrández, 1986

Larionoides foliatus Sckrank, Mingorrubio, 30TVK39, (Kulczynski, 1901), Morano \& Ferrández, 1986

Larionoides foliatus Sckrank, Navalafuente, 30TVL42, (Kulczynski, 1901), Morano \& Ferrández, 1986

Epeira cornuta (Clerck, 1758), Pinto, 30TVK57, Sanz de Diego, 1885

Distribución: Europa hasta Asia Central. Mitad sur de España.

Larinoides sclopetarius (Clerck, 1758)

- Araneus sclopetarius

CitAs DE LA CM

Larinoides sclopetarius (Clerck, 1758), Alpedrete, 30TVK10, Morano \& Ferrández, 1986

Larinoides sclopetarius (Clerck, 1758), Ambite de Tajuña, 30TVK86, Morano \& Ferrández, 1986 
Larinoides sclopetarius (Clerck, 1758), Collado Mediano, 30TVK19, Morano \& Ferrández, 1986

Larinoides sclopetarius (Clerck, 1758), Colmenar Viejo, 30TVL30, Morano \& Ferrández, 1986

Larinoides sclopetarius (Clerck, 1758), Colmenarejo. Rio Aulencia, 30TVK19, Morano \& Ferrández, 1986

Larinoides sclopetarius (Clerck, 1758), El Berrueco, 30TVL53, Morano \& Ferrández, 1986

Araneus sclopetarius (Clerck, 1758), El Escorial, 30TVK09, Fernández Galiano, 1910. Depositado en el MNCN $20.02 / 10415$

Larinoides sclopetarius (Clerck, 1758), El Pardo, 30TVK38, Morano \& Ferrández, 1986

Larinoides sclopetarius (Clerck, 1758), Embalse de Santillana, 30TVL31, Morano \& Ferrández, 1986

Larinoides sclopetarius (Clerck, 1758), Hoyo de Manzanares, 30TVK29, Morano \& Ferrández, 1986

Larinoides sclopetarius (Clerck, 1758), La Pedriza, 30TVL22, Morano \& Ferrández, 1986

Epeira sclopetaria (Clerck, 1758), Madrid, 30TVK47, Sanz de Diego, 1885

Araneus sclopetarius (Clerck, 1758), Madrid, 30TVK47, Fernández Galiano, 1910

Larinoides sclopetarius (Clerck, 1758), Madrid (capital), 30TVK47, Morano \& Ferrández, 1986

Larinoides sclopetarius (Clerck, 1758), Manzanares el Real, 30TVL31, Morano \& Ferrández, 1986

Larinoides sclopetarius (Clerck, 1758), Mingorrubio, 30TVK39, Morano \& Ferrández, 1986

Larinoides sclopetarius (Clerck, 1758), Perales de Tajuña, 30TVK65, Morano \& Ferrández, 1986

Larinoides sclopetarius (Clerck, 1758), Redueñas, 30TVL25, Morano \& Ferrández, 1986

Larinoides sclopetarius (Clerck, 1758), San Fernando de Henares, 30TVK10, Morano \& Ferrández, 1986

Larinoides sclopetarius (Clerck, 1758), Santa María de la Alameda, 30TUK99, Morano \& Ferrández, 1986

Larinoides sclopetarius (Clerck, 1758), Tielmes, 30TVK86, Morano \& Ferrández, 1986

Larinoides sclopetarius (Clerck, 1758), Titulcia, 30TVK53, Morano \& Ferrández, 1986

Larinoides sclopetarius (Clerck, 1758), Torrelodones, 30TVK29, Morano \& Ferrández, 1986

Larinoides sclopetarius (Clerck, 1758), San Agustín de Guadalix, 30TVL40, Ferrández y Fernández de Céspedes

Larinoides sclopetarius (Clerck, 1758), Valdemanco, 30TVL24, Morano \& Ferrández, 1986

Larinoides sclopetarius (Clerck, 1758), Villanueva del Pardillo, 30TVK28, Morano \& Ferrández, 1986

Distribución: Holártica. Toda la Península.

Mangora acalypha (Walckenaer, 1802)

- Aranea acalypha

CiTAS DE LA CM

Mangora acalypha (Walckenaer, 1802), Aranjuez, 30TVK53, Sanz de Diego, 1885

Mangora acalypha (Walckenaer, 1802), Aldea del Fresno, 30TUK97, Morano \& Ferrández, 1986
Mangora acalypha (Walckenaer, 1802), Colmenar Viejo, 30TVL30, Morano \& Ferrández, 1986

Mangora acalypha (Walckenaer, 1802), El Berrueco, 30TVL53, Morano \& Ferrández, 1986

Mangora acalypha (Walckenaer, 1802), El Berrueco, 30TVL52, Jiménez, 2002

Mangora acalypha (Walckenaer, 1802), El Escorial. Mte Abantos, 30TVK09, Morano \& Ferrández, 1986

Mangora acalypha (Walckenaer, 1802), El Pardo, 30TVK38, Morano \& Ferrández, 1986

Mangora acalypha (Walckenaer, 1802), Embalse de Santillana, 30TVL31, Morano \& Ferrández, 1986

Mangora acalypha (Walckenaer, 1802), Guadalix de la Sierra, 30TVL41, Morano \& Ferrández, 1986

Mangora acalypha (Walckenaer, 1802), Miraflores-Canencia. Km 2, 30TVL32, Morano \& Ferrández, 1986

Mangora acalypha (Walckenaer, 1802), Miraflores-Canencia. Km 3, 30TVL32, Morano \& Ferrández, 1986

Mangora acalypha (Walckenaer, 1802), Madrid (Ciudad Universitaria), 30TVK37, Morano \& Ferrández, 1986

Mangora acalypha (Walckenaer, 1802), Manzanares el Real, 30TVL31, Morano \& Ferrández, 1986

Mangora acalypha (Walckenaer, 1802), Mingorrubio, 30TVK39, Morano \& Ferrández, 1986

Mangora acalypha (Walckenaer, 1802), Puerto del Reventón, 30TVL32, Morano \& Ferrández, 1986

Mangora acalypha (Walckenaer, 1802), Puerto del Reventón. Arryo Jobalo, 30TVL32, Morano \& Ferrández, 1986

Mangora acalypha (Walckenaer, 1802), Robledo de Chavela, 30TUK97, Fernández Galiano, 1910. Depositado en el MNCN $20.02 / 10418$

Mangora acalypha (Walckenaer, 1802), Torrelodones, 30TVK29, Morano \& Ferrández, 1986

Mangora acalypha (Walckenaer, 1802), San Agustín de Guadalix, 30TVL40, Ferrández y Fernández de Céspedes

Mangora acalypha (Walckenaer, 1802), San Martín de Valdeiglesias, 30TUK85, Fernández Galiano, 1910. Depositado en el MNCN 20.02/10019

Mangora acalypha (Walckenaer, 1802), Vaciamadrid, 30TVK57, Morano \& Ferrández, 1986

Mangora acalypha (Walckenaer, 1802), Viñuelas, 30TVK49, Morano \& Ferrández, 1986

Distribución. Paleártica occidental. Toda la Península.

Neoscona adianta (Walckenaer, 1802)

- Aranea adianta

CITAS DE LA CM

Neoscona adianta (Walckenaer, 1802), Colmenar Viejo, 30TVL30, Morano \& Ferrández, 1986

Neoscona adianta (Walckenaer, 1802), Colmenarejo, 30TVK29, Morano \& Ferrández, 1986

Neoscona adianta (Walckenaer, 1802), El Berrueco, 30TVL53, Morano \& Ferrández, 1986

Neoscona adianta (Walckenaer, 1802), El Pardo, 30TVK38, Morano \& Ferrández, 1986

Neoscona adianta (Walckenaer, 1802), Embalse de Santillana, 30TVL31, Morano \& Ferrández, 1986

Neoscona adianta (Walckenaer, 1802), Guadalix de la Sierra, 30TVL41, Morano \& Ferrández, 1986

Neoscona adianta (Walckenaer, 1802), Hoyo de Manzanares, 30TVK29, Morano \& Ferrández, 1986 
Neoscona adianta (Walckenaer, 1802), La Pedriza, 30TVL22, Morano \& Ferrández, 1986

Neoscona adianta (Walckenaer, 1802), Mingorrubio, 30TVK39, Morano \& Ferrández, 1986

Neoscona adianta (Walckenaer, 1802), Miraflores. Canencia, 30TVL32, Morano \& Ferrández, 1986

Neoscona adianta (Walckenaer, 1802), Montarco, 30TVK57, Morano \& Ferrández, 1986

Neoscona adianta (Walckenaer, 1802), Morata deTajuña, 30TVK33, Morano \& Ferrández, 1986

Neoscona adianta (Walckenaer, 1802), Rascafría. Río Lozoya, 30TVL22, Morano \& Ferrández, 1986

Neoscona adianta (Walckenaer, 1802), Santa María de la Alameda, 30TUK99, Morano \& Ferrández, 1986

Neoscona adianta (Walckenaer, 1802), Soto del Real, 30TVL31, Morano \& Ferrández, 1986

Neoscona adianta (Walckenaer, 1802), Viñuelas, 30TVK49, Morano \& Ferrández, 1986 es la misma cita de Valdelatas de Camargo, 1982 como Araneus adiantus.

Distribución. Paleártica. Toda la Península.

Neoscona subfusca (C.L. Koch, 1837)

- Atea subfusca

CiTAs DE LA CM

Neoscona dalmatica (Doleschall, 1852), Aldea del Fresno, 30TUK97, Morano \& Ferrández, 1986

Epeira dalmatica Doleschall, 1852, Pinto, 30TVK97, Sanz de Diego, 1885

Distribucion. Europa, Asia Central y África. Sur de la Península.

Nuctenea umbratica (Clerck, 1757)

- Araneus umbraticus

CitAs DE LA CM

Nuctenea umbratica (Clerck, 1758), Lozoya, 30TVL33, Morano \& Ferrández, 1986

Nuctenea umbratica (Clerck, 1758), Montejo de la Sierra, 30TVL45, Morano \& Ferrández, 1986

Nuctenea umbratica (Clerck, 1758), Rascafría, 30TVL22, Morano \& Ferrández, 1986

Distribución. Paleártica. Norte de la Península.

Singa hamata (Clerck, 1758)

- Araneus hamatus

CitAs DE LA CM

Singa hamata (Clerck, 1758), Titulcia, 30TVK54, Morano \& Ferrández, 1986

Distribución. Paleártica. Tan solo citada de Pirineos y Guadarrama.

Zilla diodia (Walckenaer, 1802)

- Aranea diodia

CITAS DE LA CM

Epeira diodia (Walckenaer, 1802), Aranjuez, 30TVK53, Sanz de Diego, 1885

Zilla diodia (Walckenaer, 1802), Guadalix de la Sierra, 30TVL41, Morano \& Ferrández, 1986
Zilla diodia (Walckenaer, 1802), El Berrueco, 30TVL52, Jiménez, 2002

Zilla diodia (Walckenaer, 1802), Miraflores-Canencia, 30TVK32, Morano \& Ferrández, 1986

Zilla diodia (Walckenaer, 1802), Puerto del Reventón, 30TVL32, Morano \& Ferrández, 1986

Zilla diodia (Walckenaer, 1802), Torrelodones, 30TVL41, Morano \& Ferrández, 1986

Zilla diodia (Walckenaer, 1802), San Agustín de Guadalix, 30TVL40, Ferrández y Fernández de Céspedes

Zilla diodia (Walckenaer, 1802), Valdelatas, 30TVK49, Camargo, 1982

Distribución. Paleártica. Norte de la Península.

Familia Atypidae Thorell, 1870

Atypus affinis Eichwall, 1830

- Atypus affinis

Citas de LA CM

Atypus affinis Eichwall, 1830, Madrid, Blasco, 1985. Cita sin precisar la localidad.

Atypus affinis Eichwall, 1830, Miraflores, 30TVL31, Ferrández \& Beamonte, 2005

DistriBuCión. Desde gran Bretaña hasta Ucrania y el norte de África. Mitad norte de la Península.

\section{Familia Clubionidae Wagner, 1887}

Clubiona aducta Simon, 1932

- Clubiona aducta

CitAs DE LA CM

Clubiona aducta Simon, 1932, La Acebeda, 30YVL44, Ferrández \& Beamonte, 2005

Clubiona aducta Simon, 1932, Somosierra, 30TVL55, Ferrández \& Beamonte, 2005

DistribuCiÓn. Ibérica. Sistema central, desde la Sierra de Guadarrama hasta la Sierra de la Estrella y norte de Portugal.

\section{Clubiona sp}

CitAs DE LA CM

Clubiona sp, Madrid (Capital), 30TVK47, Fernández Bourgon, 2003

Familia Corinnidae Karsch, 1880

Castianeira badia (Simon, 1877)

- Agroeca badia

CITAS DE LA CM

Castianeira badia (Simon, 1877), Cantoblanco. Universidad Autonoma de Madrid, 30TVK49, Camargo \& Ferrández, 1985 Agroeca badia Simon, 1877, El Escorial, 30TVK09, Simon, 1877

Castianeira badia (Simon, 1877), Hoyo de Manzanares, 30TVK29, Camargo \& Ferrández, 1985 
DISTRIBUCION. Ibérica. Solo citada de unas cuantas provincias del centro de España. Y recientemente, de Portugal.

Familia Dictynidae O. Pickard-Cambridge, 1871

Ajmonia gratiosa (Simon, 1881)

- Dictyna gratiosa

CitAs DE LA CM

Dictyna gratiosa Simon, 1881, Robledo de Chavela, 30TUK97, Fernández Galiano, 1910. Depositado en el MNCN $20.02 / 10145$

Dictyna gratiosa Simon, 1881, San Martín de Valdeiglesias, 30TUK87, Fernández Galiano, 1910. Depositado en el MNCN 20.02/10144

DisTRIBUCIÓN. Mediterránea occidental. Solo citada del Sur de Portugal y Madrid.

\section{Familia Dysderidae C.L. Koch, 1837}

Dysdera crocata C.L. Koch, 1837*

- Dysdera crocata

CitAs DE LA CM

Dysdera crocata C.L. Koch, 1837, Alcorcón, 30TVK37, Ferrández, 1987

Dysdera crocata C.L. Koch, 1837, Algete, 30TVK59, Ferrández, 1987

Dysdera crocata C.L. Koch, 1837, Aranjuez, 30TVK53, Ferrández, 1987

Dysdera crocata C.L. Koch, 1837, Aravaca, 30TVK37, Ferrández, 1987

Dysdera crocata C.L. Koch, 1837, Brunete, 30TVK17, Ferrández, 1987

Dysdera crocata C.L. Koch, 1837, Cantoblanco, 30TVK49, Ferrández, 1987

Dysdera crocata C.L. Koch, 1837, Cercedilla, 30TVL11, Ferrández, 1987

Dysdera crocata C.L. Koch, 1837, El Escorial, 30TVK09, Ferrández, 1987

Dysdera crocata C.L. Koch, 1837, El Pardo, 30TVK38, Ferrández, 1987

Dysdera crocata C.L. Koch, 1837, Guadalix de la Sierra, 30TVL41, Ferrández, 1987

Dysdera crocata C.L. Koch, 1837, Las Rozas, 30TVL28, Ferrández, 1987

Dysdera crocata C.L. Koch, 1837, Lozoya, 30TVL33, Ferrández, 1987

Dysdera crocata C.L. Koch, 1837, Madrid, 30TVK47, Ferrández, 1987

Dysdera crocata C.L. Koch, 1837, Madrid. Aluche, 30TVK37, Ferrández, 1987

Dysdera crocata C.L. Koch, 1837, Madrid. Casa de Campo, 30TVK47, Ferrández, 1987

Dysdera crocata C.L. Koch, 1837, Madrid. Chamartin, 30TVK48, Ferrández, 1987

Dysdera crocata C.L. Koch, 1837, Madrid. Ciudad Universitaria, 30TVK37, Ferrández, 1987

Dysdera crocata C.L. Koch, 1837, Madrid. Dehesa de la Villa, 30TVK37, Ferrández, 1987
Dysdera crocata C.L. Koch, 1837, Madrid. Entrevias, 30TVK47, Ferrández, 1987

Dysdera crocata C.L. Koch, 1837, Majadahonda, 30TVL28, Ferrández, 1987

Dysdera crocata C.L. Koch, 1837, Miraflores de la Sierra, 30TVL31, Ferrández, 1987

Dysdera crocata C.L. Koch, 1837, Paracuellos del Jarama, 30TVK58, Ferrández, 1987

Dysdera crocata C.L. Koch, 1837, Pozuelo de Alarcón, 30TVK37, Ferrández, 1987

Dysdera crocata C.L. Koch, 1837, Rascafría, 30TVL22, Ferrández, 1987

Dysdera crocata C.L. Koch, 1837, Ribas del Jarama, 30TVK56, Ferrández, 1987

Dysdera crocata C.L. Koch, 1837, San Martín de Valdeglesias, 30TVK19, Ferrández, 1987

Dysdera crocata C.L. Koch, 1837, Valdetorres del Jarama, 30TVL50, Ferrández, 1987

Dysdera crocata C.L. Koch, 1837, Villalba de Guadarrama, 30TVK19, Schenckel, 1938

Dysdera crocata C.L. Koch, 1837, Villalba, 30TVK19, Ferrández, 1987

Dysdera crocata C.L. Koch, 1837, Villalba. Los Peñascales, 30TVK19, Ferrández, 1987

Dysdera crocata C.L. Koch, 1837, Villaviciosa de Odon, 30TVK25, Ferrández, 1987

DistRIBUCIÓN. Cosmopolita (antropófila). Toda la Península.

Dysdera edumifera Ferrández, 1984

- Dysdera edumifera

Citas DE LA CM

Dysdera edumifera Ferrández, 1984, Aranjuez, 30TVK53, Ferrández, 1984

Dysdera edumifera Ferrández, 1984, Valdemorillo, 30TVK10, Ferrández, 1984

Distribución. Ibérica. Conocida tan solo de unas pocas localidades de las provincias de Madrid y Albacete.

Dysdera erythrina (Walckenaer, 1802)

- Aranea erythrina

Citas DE LA CM

Dysdera erythrina (Walckenaer, 1802), Aranjuez, 30TVK53, Ferrández, 1987

Dysdera erythrina (Walckenaer, 1802), El Escorial, 30TVK09, Bolívar, 1875

Dysdera erythrina (Walckenaer, 1802), Madrid, 30TVK47, Ferrández, 1987

DisTRIBUCIÓN. Europea. En la Península ocupa fundamentalmente la zona cantábrica y pirenaica.

Dysdera gamarrae Ferrández, 1984

- Dysdera gamarrae

Citas de la CM

Dysdera gamarrae Ferrández, 1984, Alpedrete, 30TVL01, Ferrández, 1984

Dysdera gamarrae Ferrández, 1984, El Paular, 30TVL22, Ferrández, 1984

Dysdera gamarrae Ferrández, 1984, Meco. "Finca El Encin“, 30TVK87, Ferrández, 1984 
Dysdera gamarrae Ferrández, 1984, Miraflores, 30TVL31, Ferrández, 1984

Dysdera gamarrae Ferrández, 1984, Pinilla del Valle, 30TVL33, Ferrández, 1984

Dysdera gamarrae Ferrández, 1984, Valdemanco, 30TVL24, Ferrández, 1984

DISTRIBUCIÓN. Ibérica. Repartida por la mitad sur peninsular.

Harpactea fageli Brignoli, 1980

- Harpactea fageli

CiTAS DE LA CM

Harpactea fageli Brignoli, 1980, Cercedilla. El Portazgo, 30TVL11, Ferrández \& Fernández de Céspedes, 1990

Harpactea fageli Brignoli, 1980, El Escorial. La Herreria, 30TVK09, Ferrández \& Fernández de Céspedes, 1990

Harpactea fageli Brignoli, 1980, El Escorial. Silla de Felipe II, 30TVK09, Ferrández \& Fernández de Céspedes, 1990

Harpactea fageli Brignoli, 1980, Embalse de Navalmedio, 30TVL11, Ferrández \& Fernández de Céspedes, 1990

Harpactea fageli Brignoli, 1980, Embalse del Vellón, 30TVL41, Ferrández \& Fernández de Céspedes, 1990

Harpactea fageli Brignoli, 1980, Galapagar. Casa de Endrinal, 30TVK19, Ferrández \& Fernández de Céspedes, 1990

Harpactea fageli Brignoli, 1980, Guadalix de la Sierra, 30TVL41, Ferrández \& Fernández de Céspedes, 1990

Harpactea fageli Brignoli, 1980, Guadalix de la Sierra. Srra de Hoyos, 30TVL31, Ferrández \& Fernández de Céspedes, 1990

Harpactea fageli Brignoli, 1980, La Acebeda, 30TVL44, Ferrández \& Beamonte, 2005

Harpactea fageli Brignoli, 1980, La Pedriza, 30TVL22, Ferrández \& Fernández de Céspedes, 1990

Harpactea fageli Brignoli, 1980, Los Molinos, 30TVL00, Ferrández \& Fernández de Céspedes, 1990

Harpactea fageli Brignoli, 1980, Lozoya. El Chaparral, 30TVL33, Ferrández \& Fernández de Céspedes, 1990

Harpactea fageli Brignoli, 1980, Miraflores, 30TVL31, Ferrández \& Fernández de Céspedes, 1990

Harpactea fageli Brignoli, 1980, Miraflores. Dehesa de Arriba, 30TVL31, Ferrández \& Fernández de Céspedes, 1990

Harpactea fageli Brignoli, 1980, Miraflores-Pto de Canencia, 30TVL31, Ferrández \& Fernández de Céspedes, 1990

Harpactea fageli Brignoli, 1980, Navacerrada. Ladera del Chiquillo, 30TVL11, Ferrández \& Fernández de Céspedes, 1990

Harpactea fageli Brignoli, 1980, Navacerrada. Peña Pintada, 30TVL11, Ferrández \& Fernández de Céspedes, 1990

Harpactea fageli Brignoli, 1980, Piñuecar, 30TVL44, Ferrández \& Fernández de Céspedes, 1990

Harpactea fageli Brignoli, 1980, Puerto de Canencia, 30TVL32, Ferrández \& Fernández de Céspedes, 1990

Harpactea fageli Brignoli, 1980, Puerto de Malagón, 30TVL09, Ferrández \& Fernández de Céspedes, 1990

DistriBUCIÓN. Ibérica. Limitada al Sistema Central.

Harpactea serena (Simon, 1907)

- Harpactes serenus

CITAS DE LA CM

Harpactea serena (Simon, 1907), Colmenar de Oreja, 30TVK64, Ferrández \& Fernández de Céspedes, 1990
Harpactea serena (Simon, 1907), Madrid. Ciudad Universitaria, 30TVK37, Ferrández \& Fernández de Céspedes, 1990

Harpactea serena (Simon, 1907), Valdelaguna, 30TVK64, Ferrández \& Fernández de Céspedes, 1990

Distribución. Sur de Europa: Francia y España. Tan solo conocida de Madrid y Navarra.

\section{Harpactea n. sp}

Harpactea $\mathrm{n}$. sp, Colmenar Viejo, 30TVL30, Ferrández, 1987. Indica que se trata de una nueva especie pero todavía no se ha publicado su descripción.

Harpactocrates gurdus Simon, 1914

- Harpactocrates gurdus

CitAs DE LA CM

Harpactocrates gurdus Simon, 1914, Cercedilla, 30TVL11, Ferrández, 1986

Harpactocrates gurdus Simon, 1914, La Hiruela, 30TVL64, Ferrández, 1986

Harpactocrates gurdus Simon, 1914, Miraflores-Canencia. Km 4, 30TVK32, Ferrández, 1986

Harpactocrates gurdus Simon, 1914, Montejo de la Sierra, 30TVL45, Ferrández, 1986

Harpactocrates gurdus Simon, 1914, Navacerrada, 30TVL11, Ferrández, 1986

Harpactocrates gurdus Simon, 1914, Navacerrada. La Barranca, 30TVL11, Ferrández, 1986

Harpactocrates gurdus Simon, 1914, Puerto de Canencia, 30TVL32, Ferrández, 1986

Harpactocrates gurdus Simon, 1914, Puerto de la Morcuera, 30TVL31, Ferrández, 1986

Harpactocrates gurdus Simon, 1914, Puerto de Navafria, 30TVL32, Ferrández, 1986

Harpactocrates gurdus Simon, 1914, Puerto de Somosierra, 30TVL55, Ferrández, 1986

DisTRIBUCIÓN. Ibérica. Endemismo de la Sierra de Guadarrama. Las citas de Canigou, en los Pirineos franceses (Simon, 1914) no se han confirmado.

\section{Parachtes teruelis Kraus, 1955}

- Harpactocrates teruelis

Citas DE LA CM

Parachtes teruelis Kraus, 1955, Miraflores, 30TVL31, Ferrández \& Beamonte, 2005

DisTRIBUCIÓN. España.

Familia Eresidae C.L. Koch, 1837

Eresus cinnaberinus (Olivier, 1789)

- Aranea cinnaberina

CiTAS DE LA CM

Eresus cinnaberinus (Olivier, 1789), Aranjuez, 30TVK43, Sanz de Diego, 1885

Eresus frontalis Latreille, 1819, El Escorial, 30TVK09, Bolívar, 1875 
Eresus fontalis Latreille, 1819, El Escorial, 30TVK09, Simon, 1873

Eresus niger (Petagna, 1797), Pelayos, 30TUK97, Fernández Galiano, 1910

Eresus cinnaberinus (Olivier, 1789), San Agustín de Guadalix, 30TVL40, Ferrández y Fernández de Céspedes

Eresus niger (Petagna, 1787), Valdelatas, 30TVK49, Camargo, 1982

Eresus niger (Petagna, 1787), Valdelaguna, 30TVK64, Fernández de Céspedes, 1987

Distribución. Paleártica occidental. Toda la Península.

Eresus sedilloti Simon, 1881

- Eresus Sedilloti

CitAs DE LA CM

Eresus Sedilloti Simon, 1881, Aranjuez, 30TVK53, Simon, 1881

DistribuCiÓn. Ibérica. Conocida solo de Madrid y de alguna localidad de Portugal.

Stegodyphus lineatus (Latreille, 1817)

- Eresus lineatus

CiTAS DE LA CM

Stegodyphus lineatus (Latreille, 1817), Pelayos, 30TUK97, Fernández Galiano, 1910

Distribución. Mediterránea. Casi toda la Península.

\section{Eresus sp}

CitAS DE LA CM

Eresus sp, Madrid (capital), 30TVK47, Fernández Bourgon.C, 2003

Familia Filistatidae Ausserer, 1867

Filistata insidiatrix (Forskäl, 1775)

- Aranea insidiatrix

CiTAS DE LA CM

Filistata insidiatrix (Forskäl, 1775), Madrid (Capital), 30TVK47, Fernández Bourgon, 2003

Filistata insidiatrix (Forskäl, 1775), Pelayos, 30TUK97, Fernández Galiano, 1910

Filistata insidiatrix (Forskäl, 1775), San Agustín de Guadalix, 30TVL40, Ferrández y Fernández de Céspedes

Filistata insidiatrix (Forskäl, 1775), San Martín de Valdeiglesias, 30TUK85, Fernández Galiano, 1910

Filistata insidiatrix (Forskäl, 1775), Valdelaguna, 30TVK64, Fernández de Céspedes, 1987

Distribución. Mediterránea. Toda la Península Ibérica. En muchas ocasiones antropófila.

Pritha nana (Simon, 1868)

- Filistata nana

Citas DE LA CM

Phritha nana (Simon, 1868), Madrid (capital), 30TVK47, Ferrández \& Beamonte, 2005

Phritha nana (Simon, 1868), Madrid (MNCN), 30TVK47, Ferrández \& Beamonte, 2005. Depositado en el MNCN $20.02 / 12601$
Phritha nana (Simon, 1868), Torrelodones, 30TVK29, Ferrández \& Beamonte, 2005

DisTRIBUCIÓN. Mediterránea. En España tan solo hay una cita anterior de Pritha sp en Jaca (Huesca) (Blasco \& Rambla, 1985).

Familia Gnaphosidae Pocock, 1898

Callilepis concolor Simon, 1914*

- Callilepis nocturna concolor

Citas de La CM

Callilepis concolor Simon, 1914, Valdelaguna, 30TVK64, Fernández de Céspedes, 1987

Distribución. Sur de Europa. Mitad norte de la Península.

Drassodes fugax (Simon, 1878)

- Drassus fugax

Citas de la CM

Drassus fugax Simon, 1878, Aranjuez, 30TVK53, Sanz de Diego, 1885

Distribución. Paleártica. Mitad norte de la Península.

Drassodes hispanus (L. Koch, 1866)

- Drassus hispanus

Citas DE La CM

Drassodes hispanus (L. Koch, 1866), Cercedilla, 30TVL11, Depositado en el MNCN 20.02/10134. Identificado por J.A. Pérez

Drassodes hispanus (L. Koch, 1866), El Escorial, 30TVK09, Simon, 1914

Distribución. Europea. Tan solo conocida de la Sierra de Guadarrama.

Drassodes lapidosus (Walckenaer, 1802)

- Aranea lapidosa

CitAs DE LA CM

Drassodes lapidosus (Walckenaer, 1802), Pelayos, 30TUK97, Fernández Galiano, 1910. Depositado en el MNCN 20.02/10121

Drassodes lapidosus (Walckenaer, 1802), Robledo de Chavela, 30TUK97, Fernández Galiano, 1910. Depositado en el MNCN 20.02/10125

Drassodes ravidus L. Koch, Robledo de Chavela, 30TUK97, Fernández Galiano, 1910. Citado e identificado por J.A. Pérez

Drassodes lapidosus (Walckenaer, 1802), San Agustín de Guadalix, 30TVL40. Ferrández y Fernández de Céspedes

Drassodes lapidosus (Walckenaer, 1802), Valdelaguna, 30TVK64, Fernández de Céspedes, 1987

Distribución. Paleártica. Repartida por toda la Península.

Drassodes luteomicans (Simon, 1878)

- Drassus luteo-micans

Citas de la CM

Drassodes luteomicans (Simon, 1878), Cercedilla. Estación Alpina, 30TVL11, Pérez de San Román, 1947. Depositado en el MNCN 20.02/10118 
Drassodes luteomicans (Simon, 1878), Madrid, 30TVK47, Pérez de San Román, 1947. Pérez (1986) señala que uno de los ejemplares citados por Pérez de San Román, corresponde al parecer a Drassodes lutescens (C.L. Koch, 1839)

Distribución. Paleártica occidental. Mitad norte de la Península.

\section{Drassodes sp}

CitAs DE LA CM

Drassodes sp, Villaba de Guadarrama, 30TVL10, Schenckel, 1938

Gnaphosa muscorum (L. Koch, 1866)*

- Pythonissa muscorum

CitAs DE LA CM

Gnaphosa muscorum (L. Koch, 1866), El Molar, 30TVL50, Depositado en el MNCN 20.02/10120. Identificado por J.A. Pérez

DisTRIBUCIÓN. Holártica. No citada anteriormente de la Península Ibérica.

\section{Gnaphosa tigrina Simon, 1878*}

- Gnaphosa tigrina

CiTAS DE LA CM

Gnaphosa tigrina Simon, 1878, Valdelaguna, 30TVK64 Fernández de Céspedes, 1987

Distribución. Francia, Suiza y Marruecos. Citada tan solo de Granada y Teruel.

Haplodrassus dalmatensis (L. Koch, 1866)

- Drassus dalmatensis

CiTAS DE LA CM

Haplodrassus dalmatensis (L. Koch, 1866), Valdelaguna, 30TVK64, Fernández de Céspedes, 1987

DisTRIBUCIÓN. Paleártica occidental. Toda Iberia.

Haplodrassus macellinus hebes (O. Pickard-Cambridge, 1874)*

- Drassus hebes

CITAS DE LA CM

Haplodrassus hebes (O. Pickard-Cambridge, 1874), Valdelaguna, 30TVK64, Fernández de Céspedes, 1987

Distribución. Francia, Córcega y España. Haplodrassus macellinus tan solo se ha señalado una vez en la Península (Sintra-Portugal).

Haplodrassus severus (C.L. Koch, 1839)*

- Drassus severus

CitAs DE LA CM

Haplodrassus severus (C.L. Koch, 1839), Valdelaguna, 30TVK64, Fernández de Céspedes, 1987

Distribución. Mediterránea. Toda la Península Ibérica.

Haplodrassus signifer (C.L. Koch, 1839) *

- Drassus signifer

Citas DE LA CM

Haplodrassus signifer (C.L. Koch, 1839), Cercedilla. E Ventorrillo, 30TVL11, Depositado en el MNCN 20.02/10129. Identificado por J.A. Pérez.
Haplodrassus signifer (C.L. Koch, 1839), Valdelaguna, 30TVK64, Fernández de Céspedes, 1987

Distribución. Holártica. Mitad Norte de la Península Ibérica.

Leptodrassus albidus Simon, 1914*

- Leptodrassus albidus

CiTAS DE LA CM

Leptodrassus albidus Simon, 1914, Valdelaguna, 30TVK64, Fernández de Céspedes, 1987

Distribución. España e Italia. Conocida de Cataluña, Salamanca y Alicante.

\section{Micaria guttigera Simon, 1878*}

- Miicaria guttigera

Citas de LA CM

Micaria guttigera Simon, 1878, Robledo de Chavela, 30TUK97, Depositado en el MNCN 20.02/10381. Identificado por J.A. Pérez.

Distribución. Península Ibérica y Francia. Repartida por toda España.

Micaria guttulata (C.L. Koch, 1839)*

- Miacaria guttulata

CITAS DE LA CM

Micaria guttulata (C.L. Koch, 1839), Valdelaguna, 30TVK64, Fernández de Céspedes, 1987

Distribución. Europa y Asia Central. Solo citada de España (Granada, Soria y Zamora).

\section{Micaria sp}

CitAs DE LA CM

Micaria sp, Madrid (Capital), 30TVK47, Fernández Bourgon, 2003

Nomisia celerrima (Simon, 1914)*

- Pterotricha celerrima

Citas DE LA CM

Nomisia celerrima (Simon, 1914), San Agustín de Guadalix, 30TVL40, Ferrández y Fernández de Céspedes

Nomisia celerrima (Simon, 1914), Valdelaguna, 30TVK64, Fernández de Céspedes, 1987

Distribución. Francia y España. Conocida de Aragón, Andalucía y Salamanca.

Nomisia exornata (C.L. Koch, 1839)

- Pythonissa exornata

Citas de La CM

Pterotricha exornata (C.L. Koch, 1839), El Escorial, 30TVK09, Fernández Galiano, 1910

Nomisia exornata (C.L. Koch, 1839), El Pardo, 30TVK38. Depositado en el MNCN 20.02/10128. Identificado por J.A. Pérez

Melanophora lucubrans Simon, Pelayos, 30TUK97, Fernández Galiano, 1910. El material del MNCN fue revisado por Denis (1962) y consistía en un macho de Nomisia exornata. 
Nomisia exornata (C.L. Koch, 1839), San Agustín de Guadalix, 30TVL40, Ferrández y Fernández de Céspedes

Pterotricha exornata (C.L. Koch, 1839), San Martín de Valdeiglesias, 30TUK85, Fernández Galiano, 1910. Depositado en el MNCN 20.02/10136

Nomisia exornata (C.L. Koch, 1939), Valdelaguna, 30TVK64, Fernández de Céspedes, 1987

DistriBución. Paleártica occidental. Toda la Península.

Scotophaeus blackwalli (Thorell, 1871)*

- Drassus blackwalli

CiTAS DE LA CM

Scotophaeus blackwalli (Thorell, 1871), Aranjuez, 30TVK53. Depositado en el MNCN 20.02/10133. Identificado por J.A. Pérez

DistriBuCión. Cosmopolita. Centro de España y Pirineos.

Scotophaeus scutulatus (L. Koch, 1866)

- Drassus scutulatus

CitAs DE LA CM

Scotophaeus scutulatus (L. Koch, 1866), Galapagar, 30TVK19, Grimm, 1986

Distribución. Europa hasta Asia Central y Argelia. Madrid y Portugal.

Scotophaeus validus (Lucas, 1846)

- Drassus validus

CitAS DE LA CM

Drassus validus Lucas, 1846, El Escorial, 30TVK09, Pérez de San Román, 1947

Drassus validus Lucas, 1846, Madrid, 30TVK47, Pérez de San Román, 1947

Scotophaeus validus (Lucas, 1846), Madrid, 30TVK47. Depositado en el MNCN 20.02/10124. Identificado por J.A Pérez.

DistriBuCión. Mediterránea. Mitad norte de la Península.

Setaphis carmeli (O. Pickard-Cambridge, 1872)

- Zelotes carmeli

CitAs DE LA CM

Seraphis carmeli (O. Pickard-Cambridge, 1872), Torrejón de Ardoz, 30TVK67, Platnick \& Murphy, 1996. Depositado en el AMNH

DistRIBUCión. Mediterránea. Toda la Península Ibérica.

Trachyzelotes fuscipes (L. Koch, 1866)

- Melanophora fuscipes

CitAs DE LA CM

Prosthesina rubicunda (Simon, 1878), Pinto, 30TVK57, Sanz de Diego, 1885

Distribución. Europea. Citada de toda la Península.

Trachyzelotes holosericeus (Simon, 1878)

- Prosthesina holosericea

CiTAS DE LA CM

Trachyzelotes holosericeus (Simon, 1878), Torrejon de Ardoz, 30TVK67, Platnick \& Murphy, 1984. Depositado en el AMNH.
Trachyzelotes holosericeus (Simon, 1878), Valdelaguna, 30TVK64, Fernández de Céspedes, 1987

DistRIBUCión. Mediterránea occidental. Citada de la mitad Sur de la Península y de los Pirineos franceses.

Urozelotes rusticus (L. Koch, 1872)

- Prosthesina rustica

Citas DE LA CM

Urozelotes rusticus (L. Koch, 1872), Madrid (Capital), 30TVK47, Fernández Bourgon.C, 2003

Distribución. Cosmopolita. Tan solo conocida de Portugal y de las provincias de Granada y Teruel

Zelotes aeneus (Simon, 1878)*

- Prosthesina aenea

Citas de la CM

Zelotes aeneus (Simon, 1878), Valdelaguna, 30TVK64, Fernández de Céspedes, 1987

DisTRIBUCIÓN. Europea. Mitad norte de España.

Zelotes caucasicus (C.L. Koch, 1839)*

- Melanophora caucasica

Citas DE LA CM

Zelotes caucasicus (C.L. Koch, 1839), Valdelaguna, 30TVK64, Fernández de Céspedes, 1987

DisTRIBUCión. Europa y Asia Central. Cataluña, Aragón, Salamanca y Granada.

Zelotes declinans (Kulczynski, 1897)*

- Prosthesina declinans

CitAs DE LA CM

Zelotes declinans (Kulczynski, 1897), Valdelaguna, 30TVK64, Fernández de Céspedes, 1987

DistriBuCiÓN. Paleártica. Tan solo citada de las montañas de Cataluña.

Zelotes thorelli Simon, 1914

- Zelotes Thorelli

CITAS DE LA CM

Zelotes thorelli Simon, 1914, Aranjuez, 30TVK53. Depositado en el MNCN 20.02/10130. Identificado por J.A. Pérez.

Melanophora lucubrans Simon, El Escorial, 30TVK09, Fernández Galiano, 1910. El material del MNCN fue examinado por J. Denis (1962) y se trataba de Zelotes thorelli. Revisado por J.A. Pérez.

Melanophora lucubrans Simon, Robledo de Chavela, 30TUK97, Fernández Galiano, 1910. Depositado en el MNCN 20.02/10135. Actualmente etiquetado Zelotes thorelli e identificado por J.A. Pérez, corresponde al material citado por Fernández Galiano

Zelotes thorelli Simon, 1914, Valdelaguna, 30TVK64, Fernández de Céspedes, 1987

Distribución. Sur de Europa. Mitad Norte de España.

\section{Zelotes sp}

CITAS DE LA CM

Zelotes sp, Sierra de Guadarrama, Schenckel, 1938 
Familia Hersiliidae Thorell, 1869

Hersiliola maculata (Dufour, 1831)

- Aranea macullulata

CitAs DE LA CM

Hersiliola maculata (Dufour, 1831), Pantano de San Juan, 30TUK87, Ribera, Ferrández \& Pérez, 1986

Hersiliola maculata (Dufour, 1831), Presa Picadas, 30TUK96, Ribera, Ferrández \& Pérez, 1986

Distribución. Mediterránea occidental. Mitad Sur de España.

Familia Linyphiidae Blackwall, 1859

Acartauchenius nasutus (O. Pickard-Cambridge, 1879)*

- Walckenaera nasuta

CiTAS DE LA CM

Trachelocamptus nasutus (O. Pickard-Cambridge, 1879), Valdelaguna, 30TVK64, Fermández de Céspedes, 1987

DiSTRIBUCIÓN. Mediterránea occidental. Tan solo dos citas sin precisar de España y de Extremadura en Portugal.

\section{Araneoncus sp}

CitAs DE LA CM

Araneoncus sp, Valdelaguna, 30TVK64, Fernández de Céspedes, 1987

Erigone atra Blackwall, 1833*

- Erigone atra

CitAs DE LA CM

Erigone atra Blackwall, 1833, Madrid, 30TVK47. Depositado en el MNCN 20.02/10139 identificado por A. do Barros Machado

DisTRIBUCIÓN. Holártica. Solo citada de Andorra y Portugal.

Erigonoplus castellanus (O. Pickard-Cambridge, 1875)

- Erigone castellana

CITAS DE LA CM

Erigone castellana O. Pickard-Cambridge, 1875, Aranjuez, 30TVK53, O. Pickard-Cambridge, 1875

DisTRIBUCIÓN. Ibérica. Solo conocida de la localidad típica y recientemente citada de Portugal (Telfer et al., 2003).

Frontinella frutetorum (C.L. Koch, 1834)

- Linyphia frutetorum

CITAS DE LA CM

Linyphia frutetorum C.L. Koch, 1834, Pelayos, 30TUK97, Fernández Galiano, 1910. Depositado en el MNCN $20.02 / 10140$

Linyphia frutetorum C.L. Koch, 1834, Robledo de Chavela, 30TUK97, Fernández Galiano, 1910. Depositado en el MNCN 20.02/10055

Frontinella frutetorum (C.L. Koch, 1834), San Agustín de Guadalix, 30TVL40, Ferrández y Fernández de Céspedes

Distribución. Paleártica occidental. Toda la Península Ibérica.
Linyphia triangularis (Clerck, 1758)

- Araneus triangularis

CITAS DE LA CM

Linphya triangularis (Clerck, 1758), Pinto, 30TVK57, Sanz de Diego, 1885

Distribución. Paleártica. Mitad norte de la Península.

Meioneta fuscipalpis (C.L. Koch, 1836)

- Micryphantes fuscipalpis

CITAS DE LA CM

Ischnyphantes fuscipalpis (C.L. Koch, 1836), Madrid. Casa de Campo. 30TVK47, Machado, 1941. Machado (1941) indica que existen en la colección de Simon (MNHNP) ejemplares procedentes de esta localidad.

Ischnyphantes fuscipalpis (C.L. Koch, 1836), Peñalara, 30TVL12, Machado, 1941. Machado (1941) indica que existen en la colección de Simon (MNHNP) ejemplares procedentes de esta localidad.

Meioneta fuscipalpis (C.L. Koch, 1836), Valdelaguna, 30TVK64, Fernández de Céspedes, 1987

DisTRIBUCIÓN. Paleártica. Citada únicamente en España de Madrid, Granada y Segovia y en Portugal sólo de Beira litoral.

Microlinyphia pusilla (Sundevall, 1830)

- Linyphia pusilla

CITAS DE LA CM

Microlinyphia pusilla (Sundevall, 1830), Chinchón. Lgna de San Juan, 30TVK54, Ferrández \& Beamonte, 2005

Microlinyphia pusilla (Sundevall, 1830), San Agustín de Guadalix, 30TVL40, Ferrández y Fernández de Céspedes

DisTRIBUCIÓN. Holártica. Toda la península.

Oedothorax retusus (Westring, 1851)

- Erigone retusa

CiTAS DE LA CM

Gonglydium retusum (Westring, 1851), Aranjuez, 30TVK53, Simon, 1864

Gonglydium retusum (Westring, 1851), Madrid, 30TVK47, Simon, 1884

Distribución. Paleártica. Citada únicamente de Madrid y Vizcaya en España y de Beira Alta en Portugal.

Pelecopsis inedita (O. Pickard-Cambridge, 1875)*

- Erigone inedita

CITAS DE LA CM

Lophocarenum ineditum (O. Pickard-Cambridge, 1975), Valdelaguna, 30TVK64, Fernández de Céspedes, 1987

Distribución. Mediterránea (Francia e Italia). Solo citada de Portugal, en Douro litoral.

\section{Pelecospis sp}

Citas DE LA CM

Pelecopsis sp, Valdelaguna, 30TVK64, Fernández de Céspedes, 1987 
Walckenaeria corniculans (O. Pickard-Cambridge, 1875)*

- Erigone corniculans

CitAs DE LA CM

Prosopotheca corniculans (O. Pickard-Cambridge, 1875), Valdelaguna, 30TVK64, Fernández de Céspedes, 1987

Distribución: Paleártica occidental. Toda la Península.

Familia Liocranidae Lenthinen, 1967

Liocranum majus Simon, 1878

- Liocranum majus

CitAS DE LA CM

Liocranum majus Simon, 1878, Sierra de Guadarrama, Simon, 1878

Liocranum segmentatum Simon, 1878, Cercedilla. Estación alpina, 30TVL11, Peréz de San Román, 1947

Liocranum majus Simon, 1878, El Escorial, 30TVK09. Depositado en el MNCN 20.02/10378, identificado por C. Urones.

DisTRIBUCión. Francia y Península Ibérica. Toda la Península.

\section{Liocranum sp}

CitAs DE LA CM

Liocranum sp, Robledo de Chavela, 30TUK97. Depositado en el MNCN 20.02/10380. Identificado por C. Urones.

Mesiotelus mauritanicus Simon, 1909

- Mesiotelus mauritanicus

CiTAS DE LA CM

Mesiotelus mauritanicus Simon, 1909, Madrid (capital), 30TVK47, Peréz de San Román, 1947. Depositado en el MNCN 20.02/10375. Revisado por C. Urones

Mesiotelus mauritanicus Simon, 1909, Valdelaguna, 30TVK64. Fernández de Céspedes, 1987

DistRIBUCIÓN. Mediterráneo occidental. Toda la Península.

Phrurolinillus tibialis (Simon, 1878)

- Micariososma tibiale

Citas DE LA CM

Phrurolinillus tibialis (Simon, 1878), San Lorenzo del Escorial, 30TVK09, Wunderlich, 1995

DisTRIBUCIÓN. Ibérica. Solo citada de Granada y Madrid, en España y de Beira Alta, en Portugal.

Phrurolithus festivus (C.L. Koch, 1835)

- Macaria festiva

CiTAS DE LA CM

Phrurolithus festivus (C.L. Koch, 1835), La Acebeda, 30TVL44, Ferrández \& Beamonte, 2005

Phrurolithus festivus (C.L. Koch, 1835), El Escorial, 30TVK09, Schenckel, 1938

Phrurolithus festivus (C.L. Koch, 1835), Rascafría. Ladera de Cabeza mediana, 30TVL22, Urones, 1989

Distribución. Paleártica. Mitad norte de la Península Ibérica.
Phrurolithus minimus C.L. Koch, 1839*

- Phrurolithus minimus

Citas DE LA CM

Phrurolithus minimus C.L. Koch, 1839, Madrid, 30TVK47. Depositado en el MNCN 20.02/10383. Identificado por C. Urones.

Distribución. Paleártica. Conocida solo de España: Barcelona, Huesca y Léon.

\section{Familia Lycosidae Sundevall, 1833}

Alopecosa accentuata (Latreille, 1817)

- Lycosa accentuata

CITAS DE LA CM

Lycosa accentuata Latreille, 1817, Aranjuez, 30TVK53, Sanz de Diego, 1885

Distribución. Paleártica occidental. Mitad norte de la Península.

Alopecosa albofasciata (Brullé, 1832)

- Lycosa albofasciata

CitAs DE LA CM

Lycosa albofasciata Brullé, 1832, El Escorial, 30TVK09, Fernández Galiano, 1910. Depositado en el MNCN 20.02/10052, revisado por J.A. Barrientos

Alopecosa albofasciata Brullé, 1832, El Berrueco, 30TVL52, Jiménez, 2002

Lycosa albofasciata Brullé, 1832, Madrid, 30TVK47, Sanz de Diego, 1885

Lycosa albofasciata Brullé, 1832, Pinto, 30TVK57, Sanz de Diego, 1885

Alopecosa albofasciata (Brullé, 1832), San Agustín de Guadalix, 30TVL40, Ferrández y Fernández de Céspedes

Alopecosa albofasciata (Brullé, 1832), Valdelatas, 30TVK49, Camargo, 1982

Distribución. Paleártica. Toda la Península.

Alopecosa pulverulenta (Clerck, 1758)

- Araneus pulverulentus

CitAs DE LA CM

Lycosa pulverulenta (Clerck, 1758), El Escorial, 30TVK09, Fernández Galiano, 1910. Depositado en el MNCN 20.02/10049. Revisado por J.A. Barrientos.

Distribución. Paleártica. Toda la Península.

Alopecosa simoni (Thorell, 1872)

- Tarentula Simonis

Citas DE LA CM

Lycosa Simoni (Thorell, 1872), Aranjuez, 30TVK53, Sanz de Diego, 1885

Alopecosa simoni (Thorell, 1872), Montarco, 30TVK57. Depositado en el MNCN 20,02/10023. Identificado por J.A. Barrientos.

Alopecosa simoni (Thorell, 1872), Valdelaguna, 30TVK64, Fernández de Céspedes, 1987

Distribución. Europea. Citada de la mitad norte de la Península y del Algarve (Portugal). 
Arctosa cinerea (Fabricius, 1777)

- Aranea cinerea

CITAS DE LA CM

Lycosa cinerea (Fabricius, 1777), El Escorial, 30TVK09, Fernández Galiano, 1910. Depositado en el MNCN 20.02/10039. Revisado por J.A .Barrientos

Lycosa cinerea (Fabricius, 1777), Madrid, 30TVK47, Sanz de Diego, 1885

DISTRIBUCIÓN.

Paleártica. Toda la Península.

Arctosa excellens (Simon, 1876)*

- Lycosa excellens

CITAS DE LA CM

Leaena excelens (Simon, 1876), Valdelaguna, 30TVK64, Fernández de Céspedes, 1987

DisTRIBUCIÓN. Ibérica. Solo hay citas generales del Norte de España, y de forma concreta únicamente en el distrito de Porto, en Portugal.

Arctosa lacustris (Simon, 1876)*

- Lycosa lacustris

CITAS DE LA CM

Arctosa lacustris (Simon, 1876), Robledo de Chavela, 30TUK97. Depositado en el MNCN 20.02/10029. Identificado por J.A. Barrientos.

DistribuCión. Mediterránea e islas Canarias. Citada de gran parte de la Península Ibérica.

Arctosa leopardus (Sundevall, 1833)

- Lycosa leopardus

CitAs DE LA CM

Arctosa leopardus (Sundevall, 1833), Puerto de Somosierra, 30TVL55, Luna \& Delgado, 2002

DistribuCión. Paleártica. Mitad norte de la península

Arctosa perita (Latreille, 1799)

- Aranea perita

CitAs DE LA CM

Arctosa perita (Latreille, 1799), El Escorial, 30TVK09, Fernández Galiano, 1910. Depositado en el MNCN 20.02/10028. Revisado por J.A. Barrientos

Arctosa perita (Latreille, 1799), Robledo de Chavela, 30TUK97, Fernández Galiano, 1910. Depositado en el MNCN 20.02/10025. Revisado por J.A. Barrientos

DistribuCiÓn. Holártica. Toda la Península.

Arctosa personata (L. Koch, 1872)

- Lycosa personata

CITAS DE LA CM

Lycosa tomentosa Simon, 1876, Aranjuez, 30TVK53, Sanz de Diego, 1885. Barrientos et al. (1985) establecen que Lycosa tomentosa debe de corresponder a Arctosa personata.

Lycosa tomentosa Simon, 1876, Pinto, 30TVK57, Sanz de Diego, 1885
Arctosa personata (L. Koch, 1872), Valdelaguna, 30TVK64, Fernández de Céspedes, 1987

Distribución. Mediterráneo occidental. Citada de gran parte de la Península Ibérica.

Arctosa variana (C.L. Koch, 1847)*

- Arctosa variana

CitAs DE LA CM

Arctosa variana (C.L. Koch, 1847), El Molar, 30TVK50. Depositado en el MNCN 20.02/10047. Identificado por J.A. Barrientos

Arctosa variana (C.L. Koch, 1847), Madrid, 30TVK47. Depositado en el MNCN 20.01/10031; 20.02/10040 y 20.02/10111. Identificado por J.A. Barrientos.

DisTRIBUCIÓN. Mediterráneo y Asia central. Solo se conoce de Cordoba y Huesca.

Hogna radiata (Latreille, 1817)

- Lycosa radiata

CITAS DE LA CM

Lycosa radiata Latreille, 1817, Aranjuez, 30TVK53, Sanz de Diego, 1885

Lycosa radiata Latreille, 1817, El Escorial, 30TVK09, Fernández Galiano, 1910. Depositado en el MNCN 20.02/10035. Revisado por J.A. Barrientos.

Lycosa radiata Latreille, 1817, Pelayos, 30TUK97, Fernández Galiano, 1910. Depositado en el MNCN 20.02/10027. Revisado por J.A. Barrientos.

Hogna radiata (Latreille, 1817), San Agustín de Guadalix, 30TVL40, Ferrández y Fernández de Céspedes

Lycosa radiata Latreille, 1817, Valdelaguna, 30TVK64, Fernández de Céspedes, 1987

Lycosa radiata Latreille, 1817, Valdelatas, 30TVK49, Camargo, 1982

Lycosa radiata Latreille, 1817, Villaverde, 30TVK46, Sanz de Diego, 1885

Lycosa radiata Latreille, 1817, Villaviciosa de Odon, 30TVK25, Fernández Galiano, 1910. Depositado en el MNCN 20.02/10051. Revisado por J.A. Barrientos.

Distribución. Paleártica occidental. Toda la Península Ibérica.

Lycosa fasciiventris Dufour, 1835*

- Lycosa fasciiventris

CitAs DE LA CM

Lycosa tarentula fasciiventris Cercedilla, 30TVL11. Depositado en el MNCN 20.02/10038. Identificado por J.A. Barrientos

Lycosa tarentula fasciiventris El Escorial, 30TVK09. Depositado en el MNCN 20.02/10034. Identificado por J.A. Barrientos

Lycosa tarentula fasciiventris Montarco, 30TVK57. Depositado en el MNCN 20.02/10037. Identificado por J.A. Barrientos

Lycosa tarentula fasciiventris Robledo de Chavela, 30TUK97. Depositado en el MNCN 20.02/10026. Identificado por J.A. Barrientos

Lycosa fasciiventris Dufour, L, 1835, Valdelaguna, 30TVLK64, Fernández de Céspedes, 1987 
Lycosa fasciiventris Dufour, L, 1835, Valdelatas, 30TVLK49, Camargo, 1982

Distribución. Ibérica. Túnez? Toda la Península.

Pardosa amentata (Clerck, 1758)

- Araneus amentatus

Citas DE LA CM

Pardosa amentata (Clerck, 1758), Sierra de Guadarrama, Simon, 1937

Distribución. Europa. Mitad norte de la Península.

Pardosa hortenis (Thorell, 1872)

- Pardosa hortensis

Citas de La CM

Pardosa hortensis (Clerck, 1758), El Pardo, 30TVK38. Depositado en el MNCN 20.02/10044. Identificado por J.A. Barrientos

Pardosa hortensis (Clerck, 1758), Somosierra, 30TVL55, Luna \& Delgado, 2002

DistRIBUCIÓN. Paleártica. Repartida por toda la Península.

Pardosa monticola (Clerck, 1758)

- Araneus monticola

CitAs DE LA CM

Pardosa monticola (Clerck, 1758), Sierra de Guadarrama, Simon, 1937

Distribución. Paleártica. Montañas de la Península Ibérica.

Pardosa morosa (L. Koch, 1870)

- Lycosa morosa

Citas de LA CM

Pardosa morosa L. Koch, 1870, El Escorial, 30TVK09, Fernández Galiano, 1910. Depositado en el MNCN 20.02/10041. Revisado por J.A. Barrientos.

Distribución. Europa hasta Asia central. Casi toda España. No citada de Portugal.

Pardosa proxima (C.L. Koch, 1847)

- Lycosa proxima

Citas DE LA CM

Pardosa proxima (C.L. Koch, 1847), Madrid, 30TVK47. Depositado en el MNCN 20.02/10030. Identificado por J.A. Barrientos.

Pardosa proxima (C.L. Koch, 1847), Pto de Malagón, 30TUL80. Depositado en el MNCN 20.02/10045. Identificado por J.A. Barrientos.

Pardosa proxima (C.L. Koch, 1847), Valdelaguna, 30TVK64, Fernández de Céspedes, 1987

Pardosa proxima (C.L. Koch, 1847), Valdelatas, 30TVK49, Camargo, 1982

Pardosa proxima (C.L. Koch, 1847), Somosierra, 30TVL55, Luna \& Delgado, 2002

DistribuCIÓn. Paleártica occidental, incluyendo Canarias y Azores. Toda la península.
Pardosa pullata (Clerck, 1758)

- Araneus pullatus

Citas de La CM

Pardosa pullata (Clerck, 1758), Somosierra, 30TVL55, Luna \& Delgado, 2002

Distribución. Europa, Rusia y Asia Central. Mitad norte de la península.

\section{Pardosa sp}

CitAs DE LA CM

Pardosa sp, Madrid (Capital), 30TVK47, Fernández Bourgon, 2003

Pirata tenuitarsis (Simon, 1876)

- Lycosa pirata tenuitarsis

Citas DE LA CM

Lycosa pirata tenuitarsis Simon, 1876, Sierra de Guadarrama, Simon, 1937

Distribución. Europa hasta Asia central. Toda la Península Ibérica.

Trochosa ruricola (De Geer, 1778)

- Trochosa ruricola

CitAs DE LA CM

Trochosa ruricola (De Geer, 1778), Aranjuez, 30TVK53. Depositado en el MNCN 20.02/10042. Identificado por J.A. Barrientos.

Trochosa ruricola (De Geer, 1778), San Agustín de Guadalix, 30TVL40, Ferrández y Fernández de Céspedes

Trochosa ruricola (De Geer, 1778), Sierra de Guadarrama. Depositado en el MNCN 20.02/10053. Identificado por J.A. Barrientos.

Trochosa ruricola (De Geer, 1778), Somosierra, 30TVL55. Luna \& Delgado, 2002

DistRIBUCIÓN. Paleártica. Repartida por gran parte de la Península Ibérica.

\section{Trochosa sp}

CitAs DE LA CM

Trochosa sp, El Escorial, 30TVK09, Schenckel, 1938

Familia Mimetidae Simon, 1895

Ero aphana (Walckenaer, 1802)

- Aranea aphana

CitAs DE LA CM

Ero aphana (Walckenaer, 1802), Cantoblanco, 30TVK49, Ferrández \& Alberdi, 2005

Ero aphana (Walckenaer, 1802), Embalse de Santillana, 30TVL31, Ferrández \& Alberdi, 2005

Ero aphana (Walckenaer, 1802), San Agustín de Guadalix, 30TVL40, Ferrández y Fernández de Céspedes

Distribución. Paleártica occidental. Mitad norte de la Península. 
Ero furcata (Villiers, 1789)

- Aranea furcata

CITAS DE LA CM

Ero furcata (Villiers, 1789), Puerto de Morcuera, 30TVL31, Ferrández \& Alberdi, 2005

Ero furcata (Villiers, 1789), San Agustín de Guadalix, 30TVL40, Ferrández y Fernández de Céspedes

Distribución. Paleártica. Tan solo citas puntuales en España y Portugal.

Mimetus laevigatus (Keyseling, 1863)

- Ero laevigata

CiTAS DE LA CM

Mimetus laevigatus (Keyseling, 1863), Embalse de Santillana, 30TVL31, Ferrández \& Alberdi, 2005

Mimetus laevigatus (Keyseling, 1863), Nuevo Baztan, 30TVK86, Ferrández \& Alberdi, 2005

Mimetus laevigatus (Keyserling, 1863), San Agustín de Guadalix, 30TVL40, Ferrández y Fernández de Céspedes

Mimetus interfector Hentz, 1850, San Martín de Valdeiglesias, 30TUK85, Fernández Galiano, 1910. Depositado en el MNCN 20.02/10387

DistriBuCIÓN. Mediterráneo hasta Asia central. Solo citada de Madrid y Barcelona.

\section{Familia Miturgidae Simon, 1885}

Cheiracanthium mildei L. Koch, 1864

- Chiracanthium Mildeai

Citas DE LA CM

Chiracanthium Mildei L. Koch, 1864, Pinto, 30TVK57, Sanz de Diego, 1885

DisTRIBUCIÓN. Holártica y Argentina. Presente en toda la Península.

Cheiracanthium pelasgicum (C.L. Koch, 1837)*

- Bolyphantes pelagicum

CITAS DE LA CM

Cheiracanthium pelasgicum (C.L. Koch,C.L, 1837), Paracuellos del Jarama, 30TVK58. Depositado en el MNCN 20.02/10185. Determinado por C.Urones.

Distribución. Paleártica. Citada de diferentes puntos de España y Portugal, aunque sin datos de la zona norte, ni Andalucía, ni Levante.

Cheiracanthium punctorium (Villiers, 1789)*

- Aranea punctoria

CITAS DE LA CM

Cheiracanthium punctorium (Villiers, 1789), Cercedilla. Estación Alpina, 30TVL11. Depositado en el MNCN 20.02/10377. Determinado por C. Urones.

Distribución. Europa y Asia Central. Toda la Península, aunque no hay citas de Andalucia y Levante.
Cheiracanthium seidlitzi L. Koch, 1864

- Chiracanthium Seidlitzei

CITAS DE LA CM

Cheiracanthium Seidlitzei L. Koch, 1864, Aranjuez, 30TVK53, Sanz de Diego, 1885

Distribución. Paleártica occidental. Norte de la Península Ibérica.

Cheiracanthium striolatum Simon, 1878

- Chiracanthium striolatum

Citas DE LA CM

Chiracanthium striolatum Simon, 1878, Pelayos, 30TUK97, Fernández Galiano, 1910. Depositado en el MNCN 20.02/10379

Distribución. Mediterránea occidental. Norte de la Península Ibérica.

\section{Cheiracanthium sp}

CitAS DE LA CM

Chiracanthium sp?, Pinto, 30TVK57, Sanz de Diego, 1885

Familia Nemesiidae Simon, 1889

Nemesia angustata Simon, 1873*

- Nemesia angustata

CiTAS DE LA CM

Nemesia angustata Simon, 1873, Valdelaguna, 30TVK64, Fernández de Céspedes, 1987

Distribución. España. Solo existe una cita de la provincia de Málaga.

Nemesia dubia O. Pickard-Cambrige, 1874*

- Nemesia dubia

CitAs dE LA CM

Nemesia dubia O. Pickard-Cambridge, 1874, Valdelatas, 30YVK49, Camargo, 1982

DistriBuCiÓN. Mediterráneo occidental. Ampliamente repartida por la Península.

Nemesia gravieri Frade \& Bacelar, 1931

- Nemesia gravieri

CITAS DE LA CM

Nemesia gravieri Frade \& Bacelar, 1931, Sierra de Guadarrama, Schenckel, 1938

DisTRIBUCIÓN. Ibérica. Solo se conoce del Baixo Alentexo (en Portugal) y de la Sierra de Guadarrama (en España).

Nemesia hispanica L. Koch, 1871

- Nemesia hispanica

CitAs DE LA CM

Nemesia hispanica L. Koch, 1871, Madrid, 30TVK47, Bacelar, 1932

DistribuCión. España. Mitad sur de España. 
Familia Nesticidae Simon, 1894

Nesticus cellulanus (Clerck, 1758)

- Araneus cellulanus

CitAS DE LA CM

Nesticus cellulanus (Clerck, 1758), Patones. Cv del Reguerillo, 30TVL51, Machado, 1941. Depositado en el MNCN 20.02/10489; 20.02/10520; 20.02/10521 y 20.02/10522.

DisTRIBUCIÓN. Holártica. Mitad norte de la Península, falta en Portugal, donde existe otra especie Nesticus lusitanicus.

Familia Oecobiidae Blackwall,1862

Oecobius cellariorum (Dugès, 1836)

- Clotho cellariorum

Citas DE LA CM

Oecobius cellariorum (Dugès, 1836), Madrid, 30TVK47. Depositado en el MNCN 20.02/10373

Oecobius cellariorum (Dugès, 1836), Madrid, 30TVK47, Fernández Bourgon, 2003

DistriBuCiÓN. Cosmopolita. Repartida por toda la Península. Normalmente en el interior de viviendas.

Oecobius navus Blackwall, 1859

- Oecobius navus

CiTAS DE LA CM

Oecobius annulipes Lucas, 1846, Sierra de Guadarrama, Simon, 1870

DisTRIBUCión. Cosmopolita. Citas dispersas en España y Portugal.

Uroctea durandi (Walckenaer, 1809)

- Clotho Durandii

Citas DE LA CM

Uroctea Durandii (Walckenaer, 1809), Aranjuez, 30TVK53, Sanz de Diego, 1885

Uroctea durandii (Walckenaer, 1809), San Agustín de Guadalix, 30TVL40, Ferrández y Fernández de Céspedes

Uroctea Durandii (Walckenaer, 1809), Villalba de Guadarrama, 30TVK19, Schenckel, 1938

DistribuCión. Paleártica occidental. Toda la Península Ibérica.

Familia Oonopidae Simon, 1892

Oonops pulcher hispanicus Dalmas, 1916

- Oonops pulcher hispanicus

CitAs DE LA CM

Oonops pulcher hispanicus Dalmas, 1916, Peñalara. Sierra de Guadarrama, 30TVL12, Dalmas, 1916

DistriBuCiÓN. España. Tan solo conocida de la localidad tipo.
Oonops tubulatus Dalmas, 1916*

- Oonops tubulatus

CitAs DE LA CM

Oonops tubulatus Dalmas, 1916, Valdelatas, 30TVK49, Camargo, 1982

Distribución. Península ibérica y Argelia. En España solo citada de Granada.

Familia Oxyopidae Thorell, 1870

Oxyopes globifer Simon, 1876

- Oxyopes globifer

Citas DE LA CM

Oxyopes globifer Simon, 1876, Valdelatas, 30TVK49, Camargo, 1982. El material recolectado por M.Camargo fue estudiado por Barrientos (1984) quien publicó un comentario sin mencionar la localidad.

DisTRIBUCIÓN. Mediterránea hasta asia central. Solo hay citas de Madrid, Murcia y Zaragoza

Oxyopes heterophtalmus Latreille, 1804

- Oxyopes heterophtalmus

CitAS DE LA CM

Oxyopes heterophtalmus Latreille, 1804, El Escorial, 30TVK09, Fernández Galiano, 1910. Depositado en el MNCN 20.02/10360, revisado por J.A. Barrientos

Oxyopes heterophtalmus Latreille, 1804, El Berrueco, 30TVL52, Jiménez, 2002

Oxyopes heterophtalmus Latreille, 1804, Madrid, 30TVK47, Sanz de Diego, 1885

Oxyopes heterophtalmus Latreille, 1804, Montarco, 30TVK57. Depositado en el MNCN 20.02/10362, revisado por J.A. Barrientos

Oxyopes heterophtalmus Latreille, 1804, El Pardo, 30TVK38. Depositado en el MNCN 20.02/10361, revisado por J.A. Barrientos

Oxyopes heterophtalmus Latreille, 1804, Rivas-Vaciamadrid, 30TVK57. Depositado en el MNCN 20.02/10358, revisado por J.A. Barrientos

Oxyopes heterophtalmus Latreille, 1804, San Agustín de Guadalix, 30TVL40, Ferrández y Fernández de Céspedes

Oxyopes heterophtalmus Latreille, 1804, San Martín de Valdeiglesias, 30TUK85, Fernández Galiano, 1910. Depositado en el MNCN 20.02/10365, revisado por J.A. Barrientos

Oxyopes heterophtalmus Latreille, 1804, Valdelatas, 30TVK49, Camargo, 1982

Distribución. Paleártica occidental. Toda la Península.

Oxyopes lineatus Latreille, 1806

- Oxyopes lineatus

CitAs DE LA CM

Oxyopes lineatus Latreille, 1806, Aranjuez, 30TVK53, Sanz de Diego, 1885

Oxyopes lineatus Latreille, 1806, Madrid, 30TVK47, Sanz de Diego, 1885

Oxyopes lineatus Latreille, 1806, Madrid, 30TVK47, Fernández Galiano, 1910 
Oxyopes lineatus Latreille, 1806, Montarco, 30TVK57. Depositado en el MNCN 20.02/10359. Identificado por J.A. Barrientos.

Oxyopes lineatus Latreille, 1806, San Agustín de Guadalix, 30TVL40, Ferrández y Fernández de Céspedes

Oxyopes lineatus Latreille, 1806, San Martín de Valdeiglesias, 30TUK85, Fernández Galiano, 1910. Depositado en el MNCN 20.02/10364. Revisado por J.A. Barrientos

Oxyopes lineatus Latreille, 1806, Valdelatas, 30TVK49, Camargo, 1982

Distribución. Paleártica occidental. Toda la Península.

Oxyopes nigripalpis (Kulczynski, 1891)

- Oxyopes lineatus nigripalpis

Citas de LA CM

Oxyopes lineatus Latreille, 1806, Pelayos, 30TUK97, Fernández Galiano, 1910. Depositado en el MNCN 20.02/10366, revisado por J.A. Barrientos

Oxyopes lineatus nigripalpis (Kulczynski, 1891), Valdelatas, 30TVK49, Camargo, 1982

DisTRIBUCIÓN. Mediterránea. Conocida de España, y citada en las provincias de Huesca, Madrid, Salamanca y Extremadura.

\section{Oxyopes sp}

CITAS DE LA CM

Oxyopes sp, Madrid (Capital), 30TVK47, Fernández Bourgon, 2003

Oxyopes sp, Valdelaguna, 30TVK64, Fernández de Céspedes, 1987. No se pudo indenticar la especie.

\section{Familia Palpimanidae Thorell,1870}

Palpimanus gibbulus Dufour, 1820

- Palpimanus gibbulus

Citas de LA CM

Palpimanus gibbulus Dufour, 1820, Aranjuez, 30TVK53, Sanz de Diego, 1885

Palpimanus gibbulus Dufour, 1820, El Berrueco, 30TVL52, Jiménez, 2002

Palpimanus gibbulus Dufour, 1820, San Agustín de Guadalix, 30TVL40, Ferrández y Fernández de Céspedes

Palpimanus gibbulus Dufour, 1820, Valdelatas, 30TVK49, Camargo, 1982

Distribución. Paleártica occidental. Casi toda la Península Ibérica, faltando en la Cornisa Cantábrica y Pirineos.

\section{Familia Philodromidae}

Paratibellus oblongiusculus (Lucas, 1846)

- Philodromus oblongiusculus

CITAS DE LA CM

Thanatus oblongiusculus (Lucas, 1846), Madrid, 30TVK47, Fernández Galiano, 1910
Thanatus oblongiusculus (Lucas, 1846), Valdelaguna, 30TVK64, Fernández de Céspedes, 1987

Paratibellus oblongiusculus (Lucas, 1846), Valdelatas, 30TVK49, Camargo, 1982

DisTRIBUCIÓN. Europa, hasta Asia central. Citada de Castilla la Mancha, Castilla Léon, Madrid y Extremadura.

Philodromus aureolus (Clerck, 1758)

- Araneus aureolus

Citas DE LA CM

Philodromus aureolus (Clerck, 1758), El Pardo, 30TVK38, Urones, 1985. Material depositado en el MNCN 20.02/10329

Philodromus aureolus (Clerck, 1758), Navacerrada, 30TVL11, Urones, 1985. Material depositado en el MNCN 20.02/10391

Distribución. Paleártica. Toda la Península Ibérica.

Philodromus buxi Simon, 1884

- Philodromus buxi

Citas dE LA CM

Philodromus buxi Simon, 1884, Madrid. Casa de Campo. 30TVK47, Simon, 1932

DisTRIBUCIÓN. Península Ibérica y Balcanes. Mitad norte de la Penínusla.

Philodromus cespitum lividus Simon, 1875

- Philidromus lividus

Citas de LA CM

Philodromus lividus Simon, 1875, El Escorial, 30TVK09, Fernández Galiano, 1910. Material perdido (Urones, 1995)

DisTRIBUCIÓN. Europea. Centro de España.

Philodromus emarginatus (Schrank, 1803)

- Aranea emarginata

CitAs DE LA CM

Philodromus buxi Simon, 1884, San Martín de Valdeiglesias, 30TUK85, Fernández Galiano, 1910. Material depositado en el MNCN 20.02/10388 identificado incorrectamente, y revisado por Urones (1995)

Philodromus emarginatus (Schranck, 1803), El Berrueco, 30TVL52, Jiménez, 2002

DistRIBUCión. Paleártica. Toda la Península Ibérica.

Philodromus fallax Sundevall, 1833*

- Philodromus fallax

CitAs DE LA CM

Philodromus fallax Sundevall, 1833, Valdelatas, 30TVK49, Camargo, 1982

Distribución. Paleártica. Toda la Península Ibérica.

Philodromus histrio (Latreille, 1819)

- Thomisus histrio

Citas DE LA CM

Thanatus vulgaris Simon, 1870, San Martín de Valdeiglesias, 30TUK85, Fernández Galiano, 1910. Material depositado en 
el MNCN 20.02/10330. Material estudiado por Urones (1995) quien corrige la identificación previa.

DistribuCión. Holártica. Mitad norte de la Península.

Philodromus pulchellus Lucas, 1846*

- Philodromus pulchellus

Citas de La CM

Philodromus pulchellus Lucas, 1846, Valdelatas, 30TVK49, Camargo, 1982

DistRIBución. Mediterránea. Toda la Península Ibérica.

Philodromus ruficapillus Simon, 1885*

- Philodromus ruficapillus

CitAS DE LA CM

Philodromus ruficapillus Simon, 1885, Valdelatas, 30TVK49, Camargo, 1982

DistribuCión. Mediterránea occidental. Citada de Zamora, Salamanca, Cáceres y Madrid en España y de Douro Litoral en Portugal.

Philodromus rufus Walckenaer, 1826*

- Philodromus rufus

Citas de LA CM

Philodromus rufus Walckenaer, 1826, Valdelatas, 30TVK49, Camargo, 1982

Distribución. Holártica. Mitad norte de la Península y en las montañas del sur.

\section{Philodromus sp}

CITAS DE LA CM

Philodromus sp, Cercedilla estación alpina, 30TVL11. Depositado en el MNCN 20.02/10335, identificado por C. Urones

Philodromus lividus Simon, 1875, Robledo de Chavela, 30TVK97, Fernández Galiano, 1910. Depositado en el MNCN $20.02 / 10327$. Se trata de un individuo juvenil sin posibilidad de identificación Urones (1995).

Thanatus atratus Simon, 1875

- Thanatus vulgaris atratus

CitAs DE LA CM

Thanatus vulgaris atratus Simon, 1875, Sierra de Guadarrama, Schenckel, 1938

DisTRIBUCIÓN. Paleártica. Tan solo citado en la Sierra de Guadarrama.

Thanatus lineatipes Simon, 1870

- Thamatus lineatipes

CitAs DE LA CM

Thanatus lineatipes Simon, 1870, Valdeguna, 30TVK64, Fernández de Céspedes, 1987

Thanatus lineatipes Simon, 1870, Valdelatas, 30TVK49, Camargo, 1982

Thanatus striatipes Simon, 1870, Robledo de Chavela, 30TUK97, Fernández Galiano, 1910. Depositado en el MNCN 20.02/10332; estudiado por Urones (1995) quien establece su identificación correcta.
Thanatus lineatipes Simon, 1870, Sierra de Guadarrama, Simon, 1870

Distribución. Mediterránea, Mitad norte de la Península y montañas del sur.

Thanatus striatus C.L. Koch, 1845

- Thanatus striatus

CitAs DE LA CM

Thanatus striatus C.L. Koch, 1845, El Molar, 30TVK50, Urones, 1995. Material depositado en el MNCN 20.02/10229. Estudiado por C. Urones identificado previamente como Micrommata ligurinum.

DistRIBUCiÓN. Holártica. Mitad norte de España.

Thanatus vulgaris Simon, 1870

- Thanatus vulgaris

Citas de La CM

Thanatus vulgaris Simon, 1870, Montarco, 30TVK57. Material depositado en el MNCN 20.02/10201 identificado por C. Urones

Thanatus vulgaris Simon, 1870, Pelayos, 30TUK97. Material depositado en el MNCN 20.02/10392 identificado por C. Urones

Thanatus vulgaris Simon, 1870, Sierra de Guadarrama, Schenckel, 1938

Thanatus vulgaris Simon, 1870, San Agustín de Guadalix, 30TVL40, Ferrández y Fernández de Céspedes

Thanatus vulgaris Simon, 1870, Valdelaguna, 30TVK64, Urones, 1995

Thanatus vulgaris Simon, 1870, Valdelatas, 30TVK49, Urones, 1995

Distribución. Holártica. Toda la Península.

Tibellus paralellus (C.L. Koch, 1837)*

- Thanatus paralellus

Citas DE LA CM

Tibellus paralellus (C.L. Koch, 1837), Valdelatas, 30TVK49, Camargo, 1982

Distribución. Paleártica. Citada tan solo de Galicia, Huesca, Ciudad Real y Madrid.

\section{Tibellus sp}

Citas DE LA CM

Tibellus sp, El Pardo, 30TVK38. Depositado en el MNCN 20.02/10390, identificado por C. Urones.

Familia Pholcidae C.L. Koch, 1850

Holocnemus caudatus (Dufour, 1820)

- Pholcus caudatus

Citas DE La CM

Holocnemus caudatus (Dufour, 1820), Colmenar de Oreja, 30TVK64. Depositado en el MNCN 20.02/10369

Holocnemus caudatus (Dufour, 1820), Robledo de Chavela, 30TUK97, Fernández Galiano, 1910 
Holocnemus caudatus (Dufour, 1820), San Martín de Valdeiglesias, 30TUK85, Fernández Galiano, 1910. Depositado en el MNCN 20.02/10372

DistriBUCión. Iberia y Sicilia. Toda la Península Ibérica.

Holocnemus hispanicus Wienhle, 1933

- Holocnemus hispanicus

CiTAS DE LA CM

Holocnemus hispanicus Wiehle, 1933, Patones, 30TVL51, Morano.E, 2001

Holocnemus hispanicus Wiehle, 1933, Laguna de San Juan, 30TVK54, Ferrández \& Beamonte, 2005

Holocnemus hispanicus Wiehle, 1933, San Agustín de Guadalix, 30TVL40, Ferrández y Fernández de Céspedes

Distribución. Endemismo de España. Conocida solo de la mitad Sur.

Holocnemus pluchei (Scopoli, 1763)

- Aranea Pluchei

CiTAS DE LA CM

Holocnemus rivulatus Forskäl, 1775, Madrid, 30TVK47, Fernández Galiano, 1910. Depositado en el MNCN 20.02/10367

DistRIBUCIÓN. Mediterránea. Toda la Península Ibérica.

\section{Holocnemus sp}

CiTAS DE LA CM

Holocnemus sp, Madrid (Capital), 30TVK47, Fernández Bourgon, 2003

Pholcus phalangioides (Fuesslin, 1775)

- Aranea phalangioides

CITAS DE LA CM

Pholcus phalangioides (Fuesslin, 1775), Madrid, 30TVK47, Bolívar, 1875

Pholcus phalangioides (Fuesslin, 1775), Madrid (Capital), 30TVK47, Fernández Bourgon, 2003

Distribución. Cosmopolita. Toda la Península Ibérica.

Spermophora senoculata (Dugès, 1836)

- Pholcus senocukatus

CitAs DE LA CM

Spermophora senoculata (Dugès, 1836), Torrelodones, 30TVK29, Ferrández \& Beamonte, 2005

DistriBUCión. Holártica. Algunas citas repartidas por andalucia. levante, Barcelona y Zaragoza. En el interor de viviendas.

Familia Pisauridae Simon, 1897

Pisaura mirabilis (Clerck, 1758)

- Aranea mirabilis

Citas DE LA CM

Pisaura mirabilis (Clerck, 1758), El Berrueco, 30TVL52, Jiménez, 2002
Pisaura mirabilis (Clerck, 1758), El Escorial, 30TVK09, Fernández Galiano, 1910. Depositado en el MNCN 20.02/10021

Pisaura mirabilis (Clerck, 1758), El Molar, 30TVK50. Depositado en el MNCN 20.02/10020

Pisaura mirabilis (Clerck, 1758), Madrid, 30TVK47, Sanz de Diego, 1885

Pisaura mirabilis (Clerck, 1758), Madrid (Capital), 30TVK47, Fernández Bourgon, 2003

Pisaura mirabilis (Clerck, 1758), Valdelatas, 30TVK49, Camargo, 1982

DistriBución. Paleártica occidental. Toda la Península Ibérica.

Familia Salticidae Blackwall, 1841

Ballus chalybeius (Walckenaer, 1802)

- Aranea chalybeia

CITAS DE LA CM

Ballus depressus (Walckenaer, 1802), Robledo de Chavela, 30TUK97, Fernández Galiano, 1910

DistribuCión. Europa, norte de África y Asia Central. Toda la Península Ibérica.

Carrothus xantogramma (Latreille, 1819)

- Salticus xantogramma

CitAs DE LA CM

Carrothus bicolor (Walckenaer, 1802), El Escorial, 30TVK09, Simon, 1868

DistRIBUCIÓN. Paleártica occidental. Citas dispersas por la Península Ibérica.

Chalcoscirtus infimus (Simon, 1868)

- Chalcoscirtus infimus

Citas DE La CM

Chalcoscirtus infimus (Simon, 1868), Paracuellos del Jarama, 30TVK58. Depositado en el MNCN 20.02/9969. Identificado por A. do Barros Machado

Chalcoscirtus infimus (Simon, 1868), Pelayos, 30TUK97, Fernández Galiano, 1910. Depositado en el MNCN 20.02/10344

Distribución. Paleártica occidental. Citada de Portugal, Madrid y Barcelona.

Cyrba algerina (Lucas, 1846)

- Salticus algerinus

CITAS DE LA CM

Cyrba algerina (Lucas, 1846), Pelayos, 30TUK97, Fernández Galiano, 1910. Depositado en el MNCN 20.02/10351

Cyrba algerina (Lucas, 1846), Valdelaguna, 30TVK64, Fernández de Céspedes, 1987

DisTRIBUCiÓN. Paleártica occidental y Macaronésica (archipiélago canario). Mitad meridional de la Península Ibérica. 
Evarcha jucunda (Lucas, 1846)

- Salticus jucundus

CiTAS DE LA CM

Evarcha jucunda (Lucas, 1846), Robledo de Chavela, 30TUK97, Fernández Galiano, 1910

Distribución. Mediterránea. Toda la Península Ibérica.

Evarcha gambosa (Simon, 1868)

- Attus gambosus

CitAs DE LA CM

Euophrys gambosa (Simon, 1868), Pelayos, 30TUK97, Fernández Galiano, 1910. Depositado en el MNCN $20.02 / 10343$

DistRIBUCIÓN. Mediterránea. Citada tan solo de Madrid y de Navarra.

Euophrys rufibarbis (Simon, 1868)

- Attus rufibarbis

CITAS DE LA CM

Evophrys rufibarbis (Simon, 1868), Torrelaguna. Monte del reguerillo, 30TVL51, Peréz de San Román, 1947. Depositado en el MNCN 20.02/9997

Evophrys rufibarbis (Simon, 1868), Valdelaguna, 30TVK64, Fernández de Céspedes, 1987

DisTRIBUCIÓN. Paleártica occidental. Citada únicamente del Baixo Alentejo y Beira Alta en Portugal y Madrid en España.

Euophrys sulphurea (C.L. Koch, 1867)*

- Attus sulphureus

CitAs DE LA CM

Euophrys sulphurea (C.L. Koch, 1867), Montarco, 30TVK57. Material depositado en el MNCN 20.02/9993

DistribuCión. Sur de Europa y Siria. Andorra, Beira litoral y Douro litoral.

\section{Euophrys sp}

CitAs DE LA CM

Euophrys sp, El Escorial, 30TVK09. Depositado en el MNCN $20.02 / 10315$

Evophrys sp, Valdelaguna, 30TVK64. Fernández de Céspedes, 1987

Heliophanus cupreus (Walckenaer, 1802)

- Aranea cuprea

CiTAS DE LA CM

Heliophanus cupreus (Walckenaer, 1802), El Escorial, 30TVK09, Fernández Galiano, 1810

Heliophanus cupreus (Walckenaer, 1802), El Escorial, 30TVK09, Wesolowska, 1986

Heliophanus cupreus (Walckenaer, 1802), Robledo de Chavela, 30TUK97, Fernández Galiano, 1810

Distribución. Paleártica. Toda la Península Ibérica.

Heliophanus kochii Simon, 1868

- Heliophanus Kochii
CitAS DE LA CM

Heliophanus armatus Simon, 1868, Madrid, 30TVK47, Sanz de Diego, 1885

Distribución. Paleártica occidental. Toda la Península.

Heliophanus flavipes (Hahn, 1832)*

- Salticus flavipes

CitAs DE LA CM

Heliophanus flavipes (Hahn, 1832), Manzanares el Real. La Pedriza, 30TVL31. Depositado en el MNCN 20.02/10206

Distribución. Paleártica. Mitad norte de la Península.

Heliophanus lineiventris Simon, 1868

- Heliophanus lineiventris

CitAs DE LA CM

Heliophanus lineiventris Simon, 1868, San Agustín de Guadalix, 30TVL40, Ferrández y Fernández de Céspedes

Heliophanus lineiventris Simon, 1868, Sierra de Guadarrama, Simon, 1868

Distribución. Paleártica. Citada de Estremadura y Algarve, en Portugal, y de Gerona, Ciudad Real, Cáceres y Madrid en España.

Icius hamatus (C.L. Koch, 1846)*

- Marpissa hamata

CitAs DE LA CM

Icius hamatus (C.L. Koch, 1846), Madrid, 30TVK47. Material depositado en el MNCN 20.02/10352

Icius hamatus (C.L. Koch, 1846), San Martín de Valdeiglesias, 30TUK85. Material depositado en el MNCN 20.02/10353 con etiqueta antigua como I. mutabilis

Distribución. Mediterránea. Cataluña, Ciudad Real, Burgos, Badajoz y Coimbra.

Menemerus semilimbatus (Hahn, 1829)

- Attus semilimbatus

Citas de la CM

Menemerus semilimbatus (Hahn, 1829), El Escorial, 30TVK09, Fernández Galiano, 1910. Depositado en el MNCN 20.02/10349

Menemerus semilimbatus (Hahn, 1829), Madrid, 30TVK47. Depositado en el MNCN 20.02/10244

Menemerus semilimbatus (Hahn, 1829), Pinto, 30TVK57, Sanz de Diego, 1885

Menemerus semilimbatus (Hahn, 1829), San Agustín de Guadalix, 30TVL40, Ferrández y Fernández de Céspedes

Menemerus semilimbatus (Hahn, 1829), Valdaracete, 30TVK85, Fernández Galiano, 1910. Depositado en el MNCN 20.02/ 10348

DisTRIBUCión. Paleártica occidental y Argentina. Toda la Península Ibérica.

Menemerus taeniatus (L. Koch, 1867)

- Attus taeniatus

Citas DE LA CM

Menemerus taeniatus (L. Koch, 1867), Rivas-Vaciamadrid, 30TVK56, Jiménez-Valverde \& López-Colón, 2003 
DisTRIBUCIÓN. Mediterránea hasta Kazajstán, Argentina.

Pellenes arciger (Walckenaer, 1837)

- Attus arcigerus

Citas DE LA CM

Pellenes arcigerus (Walckenaer,1837), Robledo de Chavela, 30TUK97, Fernández Galiano, 1910. Depositado en el MNCN 20.02/10342

Pellenes semi-ater Simon, 1868, Sierra de Guadarrama, Simon, 1876

Distribución. Mediterránea. Casi toda España, falta en Portugal.

Pellenes brevis Simon, 1868

- Attus brevis

CitAs dE LA CM

Attus brevis Simon, 1868, Sierra de Guadarrama, Simon, 1868

Distribución. Mediterránea occidental. Solo citada de Madrid y Barcelona.

Pellenes nigrociliatus (Simon, 1876)*

- Attus nigrociliatus

Citas de LA CM

Pellenes nigrociliatus (Simon, 1876), Valdelaguna, 30TVK64, Fernández de Céspedes, 1987

Distribución. Paleártica. Citada de Huesca y Portugal (sin precisar localidad).

Philaeus chysops (Poda, 1761)

- Aranea chrysops

CITAS DE LA CM

Philaeus chrysops (Poda, 1761), Madrid, 30TVK47, Sanz de Diego, 1885

Philaeus chrysops (Poda, 1761), El Escorial, 30TVK09, Fernández Galiano, 1910. Depositado en el MNCN 20.02/10341

Philaeus chrysops (Poda, 1761), Galapagar, 30TVK19. Depositado en el MNCN 20.02/9994

Philaeus chrysops (Poda, 1761), Robled de Chavela, 30TUK97, Fernández Galiano, 1910. Depositado en el MNCN 20.02/10357

Distribución. Paleártica occidental. Mitad norte de la Península.

Phlegra bresnieri (Lucas, 1846)

- Attus Bresnieri

CITAS DE LA CM

Phlegra Bresnieri (Lucas, 1846), El Escorial, 30TVK09, Fernández Galiano, 1910. Depositado en el MNCN 20.02/10347

Phlegra Bresnieri (Lucas, 1846), Robledo de Chavela, 30YUK97, Fernández Galiano, 1910. Depositado en el MNCN 20.02/10346

Distribución. Paleártica occidental. Toda la Península Ibérica.

\section{Phlegra fasciata (Hahn.1826)*}

- Attus fasciatus

Citas de LA CM

Phlegra fasciata (Hahn, 1826), Valdelguna, 30TVK64, Fernández de Céspedes, 1987

Distribución. Holártica. Conocida de Huesca, Córdoba y Zaragoza,

\section{Phlegra sp}

CITAS DE LA CM

Phlegra sp, Valdelaguna, 30TVK64, Fernández de Céspedes, 1987

Saites barbipes (Simon, 1868)

- Attus barbipes

Citas dE LA CM

Saites barbipes (Simon, 1868), El Escorial, 30TVK09, Simon, 1868

DISTRIBUCIÓN. Mediterránea, introducida en Europa

Salticus scenicus (Clerck, 1758)

- Salticus scenicus

Citas dE LA CM

Salticus scenicus (Clerck, 1758), Torrelodones, 30TVK29, Ferrández \& Beamonte, 2005

Salticus scenicus (Clerck, 1758), San Agustín de Guadalix, 30TVL40, Ferrández y Fernández de Céspedes

DisTRIBUCión. Holártica. Toda la península.

\section{Salticus sp}

CitAS DE LA CM

Salticus sp, Aranjuez, 30TVK53. Material depositado en el MNCN 20.02/10340

Sibianor aurocinctus tantalus (Ohlert, 1865)

- Heliophanus auratus tantalus

CitAs DE LA CM

Bianor tantalus (Ohlert, 1865), El Escorial, 30TVK09, Simon, 1868

Distribución. Paleártico. Conocida de las provincias de Jaén, Huesca y Madrid.

Synageles albotrimaculatus (Lucas, 1846)

- Salticus albotrimaculatus

Citas dE LA CM

Synageles albotrimaculatus (Lucas, 1846), Laguna de San Juan, 30VK54, Jiménez-Valverde \& Wesolowska, 2005

DisTRIBUCIÓN. Mediterráneo occidental. En España tan solo una cita de Madrid.

Familia Segestriidae Simon, 1893

Segestria florentina (Rossi, 1790)

- Aranea florentina

CitAs DE LA CM

Segestria florentina (Rossi, 1790), El Escorial, 30TVK09, Fernández Galiano, 1910 
Distribución. Paleártica occidental. Mitad norte de la Península.

Segestria senoculata (Linnaeus, 1758)

- Aranea senoculata

CitAs DE LA CM

Segestria senoculata (Linnaeus, 1758), Puerto de Cotos. P.N de Peñalara, 30TVL12, Ferrández \& Beamonte, 2005

DistriBuCión. Paleártica. Mitad norte de la península

\section{Segestria sp}

CiTAS DE LA CM

Segestria sp, Valdelatas, 30TVK49, Camargo, 1982

Familia Scytodiidae Blackwall,1852

Scytodes berthelothi Lucas, 1839

- Scytodes Bertheloti

CITAS DE LA CM

Scytodes bertheloti Lucas, 1839, Pinto, 30TVK57, Sanz de Diego, 1885

DisTRIBUCIÓn. Sur del Mediterráneo, Canarias y Senegal. Citada de Zaragoza?, Valencia, Madrid y Sevilla.

Scytodes thoracica (Latreille, 1804)

- Aranea thoracica

CiTAS DE LA CM

Scytodes thoracica (Latreille, 1804), Madrid (Capital), 30TVK47, Fernández Bourgon, 2003

Scytodes thoracica (Latreille, 1804), Pelayos, 30TUK97, Fernández Galiano, 1910. Depositado en el MNCN $20.02 / 10000$

Scytodes thoracica (Latreille, 1804), Robledo de Chavela 30TUK97, Fernández Galiano, 1910. Depositado en el MNCN 20.02/9970

DistriBUCión. Holártica y también en las Islas del Pacifico. Toda la Península Ibérica (en el interior de las casas en muchas ocasiones).

Scytodes velutina Heineken \& Lowe, 1835*

- Scytodes velutina

Citas DE LA CM

Scytodes velutina delicatula Simon, 1873, Valdelatas, 30TVK49, Camargo, 1982

Scytodes velutina delicatula Simon, 1873, San Martín de la Vega, 30TVK55. Material depositado en el MNCN 20.02/9999

DisTRIBUCIÓN. Mediterránea. Citada de toda la península ibérica.

Familia Sicariidae Keyserling, 1880

Loxosceles rufescens (Dufour, 1820)

- Scytodes rufescens

CitAs DE LA CM

Loxosceles rufescens (Dufour, 1820), Madrid, 30TVK47. Material depositado en el MNCN 20.02/9998
Loxosceles rufescens (Dufour, 1820), Madrid (Capital), 30TVK47, Fernández Bourgon, 2003

Loxosceles rufescens (Dufour, 1820), Robledo de Chavela 30TUK97, Fernández Galiano, 1910. Depositado en el MNCN $20.02 / 10001$

Distribución. Cosmopolita. Toda la Península Ibérica, en muchas ocasiones en el interior de viviendas.

Familia Sparassidae Bertkau,1872

Eusparassus dufouri Simon, 1932*

- Eusparassus Dufouri

Citas de La CM

Eusparassus dufouri Simon, 1932, Patones. Mte del Reguerillo, 30TVL51. Material depositado en el MNCN 20.02/9996. Identificado por C. Urones.

Eusparassus dufouri Simon, 1932, San Agustín de Guadalix, 30TVL40, Ferrández

DisTRIBUCIÓN. Mediterránea occidental. Tan solo falta en la cornisa cantábrica.

Micrommata ligurina (C.L. Koch, 1845)

- Sparassus ligurinus

Citas de la CM

Micrommata ligurina (C.L. Koch, 1845), Alpedrete, 30TVK10, Urones, 2004

Micrommata ligurina (C.L. Koch, 1845), Aranjuez, 30TVK53, Sanz de Diego, 1885

Micrommata ligurina (C.L. Koch, 1845), Aranjuez, 30TVK53, Urones, 2004

Micrommata ligurina (C.L. Koch, 1845), Cerceda, 30TVL20, Urones, 2004

Micrommata ligurina (C.L. Koch, 1845), Cercedilla, 30TVL11, Urones. C, 2004

Micrommata ligurina (C.L. Koch, 1845), El Escorial, 30TVK09, Fernández Galiano, 1910

Micrommata ligurina (C.L. Koch, 1845), El Escorial, 30TVK09, Urones, 2004

Micrommata ligurina (C.L. Koch, 1845), Galapagar, 30TVK19, Sanz de Diego, 1885

Micrommata ligurina (C.L. Koch, 1845), Leganes, 30TVK36, Urones, 2004

Micrommata ligurina (C.L. Koch, 1845), Manzanares el Real, 30TVL31, Urones, 2004

Micrommata ligurina (C.L. Koch, 1845), Pinilla de Buitrago, 30TVL43, Urones, 2004

Micrommata ligurina (C.L. Koch, 1845), Rivas del Jarama, 30TVK56, Urones, 2004

Micrommata ligurina (C.L. Koch, 1845), San Fernando de Henares, 30TVK57, Urones, 2004

Micrommata ligurina (C.L. Koch, 1845), Valdelatas, 30TVK49, Camargo, 1982

Distribución. Mediterráneo hasta Asia Central. Toda la Península Ibérica.

Micrommata virescens (Clerck, 1758)

- Araneus virenscens

Citas de la CM

Micrommata virescens (Clerck, 1758), Madrid, 30TVK47, Urones, 2004 
Micrommata virescens (Clerck, 1758), Paracuellos del Jarama, 30TVK58, Urones, 2004. Depositado en el MNCN 20.02/10009

DistriBuCIÓN. Paleártica. Repartida por toda la Península, aunque falta en Levante y parte de Andalucia.

\section{Micrommata sp}

CiTAS DE LA CM

Micrommata sp, Cercedilla. estación alpina, 30TVL11. Material depositado en el MNCN 20.02/10325. Identificado por C. Urones.

Olios argelasius (Walckenaer, 1805)

- Sparassus argelasius

CiTAS DE LA CM

Olios spongitarsis Dufour, 1820, Robledo de Chavela, 30TUK97, Fernández Galiano, 1910

DistriBuCión. Mediterráneo y Canarias. Toda la Península Ibérica.

\section{Familia Tetragnathidae Menge, 1866}

\section{Metellina merianae (Scopoli, 1763)}

- Aranea Merianae

CITAS DE LA CM

Meta Merianae (Scopoli, 1763), El Escorial, 30TVK09, Fernández Galiano, 1910. Depositado en el MNCN $20.02 / 10425$

Meta Merianae (Scopoli, 1763), El Escorial, 30TVK09, Schenckel, 1938

Meta merianae (Scopoli, 1763), Guadalix de la Sierra, 30TVL41, Morano, 1984

Meta merianae (Scopoli, 1763), Miraflores-Canencia, 30TVL32, Morano, 1984

Meta merianae (Scopoli, 1763), Pto de Canencia, 30TVL33, Morano, 1984

DistriBución. Paleártica occidental. Toda la Península Ibérica, falta en Levante.

Meta segmentata (Clerck, 1758)

- Araneus segmentatus

CiTAS DE LA CM

Meta segmentata (Clerck, 1758), Torrelaguna. $\mathrm{Cv}$ del Reguerillo, 30TVL52, Morano, 1984

Distribución. Paleártica, introducida en Canadá. Toda la Península Ibérica.

Pachygnatha clercki Sundevall, 1829*

- Pachygnatha clercki

Citas DE LA CM

Pachygnatha clercki Sundevall, 1929, San Fernando de Henares, 30TVK68, Morano, 1984

DistribuCión. Holártica. Mitad norte de la Península.

Pachygnatha simoni Senglet, 1972

- Pachygnatha simoni

CitAs DE LA CM

Pachygnatha simoni Senglet, 1972, Puerto de la Morcuera, 30TVL31, Morano, 1984
Pachygnatha simoni Senglet, 1972, Puerto de Navafria, 30TVL32, Morano, 1984

DisTRIBUCIÓN. España. Sistema central y sistema Ibérico.

Tetragnatha extensa (Linnaeus, 1758)

- Aranea extensa

CitAs DE LA CM

Tetragnatha extensa (Linnaeus, 1758), Alpedrete, 30TVK10, Morano, 1984

Tetragnatha extensa (Linnaeus, 1758), Arganda-Morata de Tajuña, 30TVK65, Morano, 1984

Tetragnatha extensa (Linnaeus, 1758), Arganda. Pte de Arganda, 30TVK55, Morano, 1984

Tetragnatha extensa (Linnaeus, 1758), El Escorial, 30TVK09, Fernández Galiano, 1910. Depositado en el MNCN 20.02/10435

Tetragnatha extensa (Linnaeus, 1758), El Pardo, 30TVK38, Morano, 1984

Tetragnatha extensa (Linnaeus, 1758), Guadalix. Embalse del Vellón, 30TVL31, Morano, 1984

Tetragnatha extensa (Linnaeus, 1758), Manzanares el Real, 30TVL31, Morano, 1984

Tetragnatha extensa (Linnaeus, 1758), Mingorrubio, 30TVK39, Morano, 1984

Tetragnatha extensa (Linnaeus, 1758), Montarco, 30TVK57, Morano, 1984

Tetragnatha extensa (Linnaeus, 1758), Pinto, 30TVK57, Fernández Galiano, 1910

Tetragnatha extensa (Linnaeus, 1758), Robledo de Chavela, 30TUK97, Fernández Galiano, 1910. Depositado en el MNCN 20.02/10440

Tetragnatha extensa (Linnaeus, 1758), San Fernando de Henares, 30TVK68, Morano, 1984

Tetragnatha extensa (Linnaeus, 1758), San Martín de Valdeiglesias, 30TUK85, Fernández Galiano, 1910. Depositado en el MNCN 20.02/10442

Tetragnatha extensa (Linnaeus, 1758), Torrelodones, 30TVK29, Morano, 1984

DistriBución. Holártica. Toda la Península Ibérica.

Tetragnatha obtusa C.L. Koch, 1837

- Tetragnatha obtusa

CITAS DE LA CM

Tetrahgnatha obtusa C.L. Koch, 1837, Guadalix de la Sierra. Emb del Vellón, 30TVL31, Morano, 1984

Tetragnatha chrysoclora Audouin, Pinto, 30TVK57, Sanz de Diego, 1885

DistRIBUCIÓN. Paleártica. Distribuida por toda la Península.

Familia Theridiidae Sundevall,1833

Archaearanea tepidariorum (C.L. Koch, 1841)

- Theridion tepidariorum

CitAs DE LA CM

Archaearanea tepidariorum (C.L. Koch, 1841), La Acebeda, 30TVL44, Ferrández \& Beamonte, 2005

Distribución. Cosmopolita. Conocida de Cataluña, Zaragoza, Cádiz y también de Portugal 
Dipoena sp

CiTAS DE LA CM

Dipoena sp, Madrid (Capital), 30TVK47, Fernández Bourgon, 2003

Enoplognatha ovata (Clerck, 1758)

- Araneus ovatum

CiTAS DE LA CM

Enoplognatha ovata (Clerck, 1758), San Agustín de Guadalix, 30TVL40, Ferrández y Fernández de Céspedes

Theridion lineatum (Clerck, 1758), San Martín de Valdeiglesias, 30TUK85, Fernández Galiano, 1910. Depositado en el MNCN 20.02/9972

DistriBución. Holártica. Toda la Península Ibérica.

\section{Enoplognatha sp}

CITAS DE LA CM

Enoplognatha sp, Valdelaguna, 30TVK64, Fernández de Céspedes, 1987

Episinus truncatus Latreille, 1809

- Episinus truncatus

Citas DE LA CM

Episinus truncatus Latreille, 1873, Loeches, 30TVK67, Fernández Galiano, 1910. Depositado en el MNCN 20.02/9984

DistRIBUCIÓn. Paleártica occidental. Citada de Cataluña, Pontevedra y Madrid, en España y del Bajo Alentejo y Extremadura, en Portugal.

Latrodectes tredecimguttatus (Rossi, 1790)*

- Aranea tredecimguttata

CiTAS DE LA CM

Latrodectes tredecimguttatus (Rossi, 1790), Valdenoceda. Santa Catalina, 30TUK97. Material depositado en el MNCN 20.02/9977. Material identificado por A. de Barros Machado.

Distribución. Mediterráneo hasta Asia Central. Presente en gran aparte de la Península, si bien no hay citas de una gran parte de las provincias del interior

Steatoda albomaculata (De Geer, 1778)

- Lithyphantes albomaculatus

CITAS DE LA CM

Lithyphantes corollatus (C.L. Koch, 1837), San Martín de Valdeiglesias, 30YUK85, Fernández Galiano, 1910 Depositado en el MNCN 20.02/9987

DistriBuCión. Cosmopolita. Mitad norte de la Península Ibérica.

Steatoda grossa (C.L. Koch, 1838)

- Theridium grossum

Citas DE LA CM

Steatoda grossa (C.L. Koch, 1838), Madrid (Capital), 30TVK47, Fernández Bourgon, 2003

Teutana grossa (C.L. Koch, 1838), Pinto, 30TVK57, Sanz de Diego, 1885

DistriBución. Cosmopolita. Toda la Península.
Staetoda paykullana (Walckenaer, 1805)

- Lithyphantes paykullanus

CITAS DE LA CM

Lithyphantes Paykullanus Walckenaer, 1805, Pinto, 30TVK57, Sanz de Diego, 1885

Steatoda paykullana (Walckenaer, 1805), San Agustín de Guadalix, 30TVL40, Ferrández y Fernández de Céspedes

Distribución. Paleártica occidental. Toda la Península Ibérica.

Steatoda phalerata (Panzer, 1801)

- Phalangium phaleratum

CitAs DE LA CM

Asagena phalerata (Panzer, 1801), Aranjuez, 30TVK53, Sanz de Diego, 1885

Asagena phalerata (Panzer, 1801), Robledo de Chavela, 30TUK97, Fernández Galiano, 1910

Steatoda phalerata (Panzer, 1801), Valdelaguna, 30TVK64, Fernández de Céspedes, 1987

Distribución. Paleártica occidental. Mitad norte de la Península Ibérica.

Staetoda triangulosa (Walckenaer, 1802)

- Aranea triangulosa

Citas DE LA CM

Steatoda triangulosa (Walckenaer, 1802), Madrid (Capital), 30TVK47, Fernández Bourgon, 2003

Teutana triangulosa (Walckenaer, 1802), Robledo de Chavela, 30TUK97, Fernández Galiano, 1910

Distribución. Cosmopolita, Toda la Península Ibérica.

Theridion impressum L. Koch, 1881*

- Theridion impressum

Citas de La CM

Theridion impressum L. Koch, 1881, Montarco, 30TVK57. Depositado en el MNCN 20.02/9986

DistRIBUCIÓN. Holártica. Repartida por gran parte de España.

Theridion pictum (Walckenaer, 1802)

- Aranea picta

CitAs DE LA CM

Theridion pictum (Walckenaer, 1802), Robledo de Chavela, 30TUK97, Fernández Galiano, 1910. Depositado en el MNCN 20.02/9971

DistribuCión. Holártica. Solo citada de Madrid.

Familia Thomisidae Sundevall,1833

\section{Coriarachne sp}

CITAS DE LA CM

Coriarachne depressa C.L. Koch, 1866, Robledo de Chavela, 30TVK97, Fernández Galiano, 1910. Depositado en el MNCN 20.02/10502. Revisado por C. Urones 
Heriaeus mellottei Simon, 1866*

- Heriaeus mellottei

CitAs dE LA CM

Heriaeus mellottei Simon, 1866, Cercedilla. Estación alpina, 30TVL11. Material depositado en el MNCN 20.02/10515. Identificado por C. Urones

Heriaeus oblongus Simon, 1918, Valdelatas, 30TVK49, Camargo, 1982

Distribución. Paleártica. Distribuida por toda la Península Ibérica.

\section{Heriaeus sp}

Citas DE LA CM

Heriaeus sp, El Pardo, 30TVK38. Material depositado en el MNCN 20.02/11669. Identificado por C. Urones

Heriaeus hirsutus Walckenaer, Pelayos, 30TUK97, Fernández Galiano, 1910. Depositado en el MNCN 20.02/10014. Estudiado por C. Urones

Heriaeus hirsutus Walckenaer, Robledo de Chavela, 30TUK97, Fernández Galiano, 1910. Depositado en el MNCN 20.02/10013. Estudiado por C. Urones

Misumena vatia (Clerck, 1758)

- Araneus vatius

CITAS DE LA CM

Misumena vatia (Clerck, 1758), Pelayos, 30TUK97, Fernández Galiano, 1910. Depositado en el MNCN 20.02/10012. Estudiado por C. Urones

Misumena vatia (Clerck, 1758), Robledo de Chavela, 30TUK97, Fernández Galiano, 1910. Depositado en el MNCN 20.02/10006. Estudiado por C. Urones

DisTRIBUCIÓN. Holártica. Toda la Península Ibérica.

Ozyptila bicuspis Simon, 1932*

- Oxyptila bicuspis

CiTAS DE LA CM

Oxyptila bicuspis Simon, 1932, Valdelatas, 30TVK49, Camargo, 1982

DisTRIBUCIÓN. Francia y España. Mitad norte de España.

Ozyptila blackwalli Simon, 1875*

- Oxyptila Blackwalli

CiTAS DE LA CM

Ozyptila blackwalli Simon, 1875, Valdelatas, 30TVK49, Camargo, 1982

Distribución. Paleártica. Solo citada de Cataluña.

Ozyptila pauxilla (Simon, 1870)

- Thomisus pauxillus

CiTAS DE LA CM

Ozyptila pauxilla (Simon, 1870), El Berrueco, 30TVL52, Jiménez, 2002

Oxyptila nigella Simon, 1875, Valdelatas, 30TVK49, Camargo, 1982

DistribuCiÓn. Mediterráneo occidental. Ciudad Real, Barcelona y Cáceres, en España y Baixo Alentejo y Douro litoral, en Portugal
Ozyptila perplexa Simon, 1875

- Oxyptila perplexa

CitAS DE LA CM

Oxyptila perplexa Simon, 1875, Aranjuez, 30TVK53, Simon, 1875

Distribución. Mediterráneo occidental. Sólo citada de Madrid y de los Pirineos.

Ozyptila umbraculorum Simon, 1932

- Oxyptila umbraculorum

CitAS DE LA CM

Ozyptila umbraculorum Simon, 1932, El Berrueco, 30TVL52, Jiménez, 2002

Distribución. Ibérica. Citada de los Pirineos orientales, de Portugal: Alentejo y Douro litoral y en España de Granada y Cáceres.

Pistius truncatus (Pallas, 1772)

- Aranea truncata

CitAs DE LA CM

Pistius truncatus (Pallas, 1772), El Berrueco, 30TVL52, Jiménez, 2002

Pistius truncatus (Pallas, 1772), Madrid, 30TVK47. Material depositado en el MNCN 20.02/10498. Identificado por C. Urones.

Distribución. Paleártica. Conocida de gran parte de España y Portugal.

Runcinia grammica (C.L. Koch, 1837)

- Xysticus grammicus

CitAs DE LA CM

Runcinia grammica (C.L. Koch, 1837), El Berrueco, 30TVL52, Jiménez, 2002

Runcinia lateralis (C.L. Koch, 1838), Loeches, 30TVK67, Fernández Galiano, 1910. Depositado en el MNCN 20.02/10011. Revisado por C. Urones

Runcinia lateralis (C.L. Koch, 1838), Madrid, 30TVK47, Fernández Galiano, 1910. Depositado en el MNCN 20.02/10509. Revisado por C. Urones

Runcinia lateralis (C.L. Koch, 1838), San Martín de Valdeiglesias, 30TUK85, Fernández Galiano, 1910. Depositado en el MNCN 20.02/10005. Revisado por C. Urones

Runcinia lateralis (C.L. Koch, 1838), Valdelatas, 30TVK49, Camargo, 1982

Distribución. Paleártica, Santa Helena y sur de África. Toda la Península Ibérica.

Synaema globossum (Fabricius, 1775)

- Aranea globosa

CitAS DE LA CM

Synaema globosa (Fabricius, 1775), Aranjuez, 30TVK53, Sanz de Diego, 1885

Synaema globosum (Fabricius, 1775), El Berrueco, 30TVL52, Jiménez, 2002

Synaema globosum (Fabricius, 1775), El Escorial, 30TVK09, Fernández Galiano, 1910 
Synaema globosum (Fabricius, 1775), Madrid, 30TVK47, Fernández Galiano, 1910. Depositado en el MNCN 20.02/10003. Revisado por C. Urones

Synaema globosum (Fabricius, 1775), Montarco, 30TVK57. Depositado en el MNCN 20.02/11668. Identificado por C. Urones

Synaema globosum (Fabricius, 1775), Pelayos, 30TUK97, Fernández Galiano, 1910. Depositado en el MNCN 20.02/10507. Revisado por C. Urones

Synaema globosum (Fabricius, 1775), Pinto, 30TVK57, Sanz de Diego, 1885

Synaema globosum (Fabricius, 1775), Robledo de Chavela, 30TUK97, Fernández Galiano, 1910. Depositado en el MNCN 20.02/10007. Revisado por C. Urones

Synaema globosum (Fabricius, 1775), San Agustín de Guadalix, 30TVL40, Ferrández y Fernández de Céspedes

Synaema globosum (Fabricius, 1775), San Martín de Valdeiglesias, 30TUK85, Fernández Galiano, 1910. Depositado en el MNCN 20.02/10506. Revisado por C.Urones

Synaema globosum (Fabricius, 1775), Valdelatas, 30TVK49, Camargo, 1982

Distribución. Paleártica occidental. Toda la Península Ibérica.

Thomisus onustus Walckenaer, 1805

- Thomisus onustus

Citas DE LA CM

Thomisus onustus Walckenaer, 1805, Aranjuez, 30TVK53, Sanz de Diego, 1885

Thomisus onustus Walckenaer, 1805, El Berrueco, 30TVL52, Jiménez, 2002

Thomisus onustus Walckenaer, 1805, El Escorial, 30TVK09, Fernández Galiano, 1910

Thomisus onustus Walckenaer, 1805, Madrid, 30TVK47, Sanz de Diego, 1885

Thomisus albus Gmelin, 1789, Montarco, 30TVK57. Materia depositado en el MNCN 20.02/ 10004. Identificado por C. Urones.

Thomisus onustus Walckenaer, 1805, Montarco, 30TVK57. Depositado en el MNCN 20.02/10008. Revisado por C. Urones.

Thomisus albus Gmelin, 1789, Robledo de Chavela, 30TUK97, Fernández Galiano, 1910. Depositado en el MNCN 20.02/10513. Revisado por C. Urones.

Thomisus onustus Walckenaer, 1805, San Agustín de Guadalix, 30TVL40, Ferrández y Fernández de Céspedes

Thomisus onustus Walckenaer, 1805, San Martín de Valdeiglesias, 30TUK85, Fernández Galiano, 1910. Depositado en el MNCN 20.02/10492. Revisado por C. Urones.

Thomisus onustus Walckenaer, 1805, Valdelatas, 30TVK49, Camargo, 1982

Distribución. Paleártica. Toda la Península Ibérica.

Tmarus piochardi Simon, 1866

- Tmarus piochardi

CiTAS DE LA CM

Tmarus piochardi Simon, 1866, Madrid, 30TVK47, Fernández Galiano, 1910

Distribución. Europa, Norte de África y Yemen. Toda la Península Ibérica.
Tmarus staintoni (O. Pickard-Cambridge, 1873)

- Monaeses Staintoni

CitAs DE LA CM

Tmarus staintoni (Pickard-Cambridge, 1873), Madrid. Casa de Campo, 30TVK47, Simon, 1932

Distribución. Mediterránea occidental. Citada solo de Cáceres, Madrid y Salamanca, falta en Portugal.

\section{Tmarus sp}

Citas de la CM

Tmarus sp, El Pardo, 30TVK38. Material depositado en el MNCN 20.02/10491. Estudiado por C. Urones

Tmarus sp, Madrid, 30TVK47. Material depositado en el MNCN 20.02/10516. Estudiado por C. Urones

Xysticus acerbus Thorell, 1872

- Xysticus acerbus

Citas DE LA CM

Xysticus acerbus Thorell, 1872, El Escorial, 30TVK09, Fernández Galiano, 1910. Depositado en el MNCN 20.02/10479. Revisado por C. Urones.

Xysticus acerbus Thorell, 1872, Madrid, 30TVK47. Material depositado en el MNCN 20.02/10516. Identificado por C. Urones

Distribución. Europa y Asia Central. Mitad norte de la Península.

Xysticus audax (Schranck, 1803)

- Aranea audax

CitAS DE LA CM

Xysticus pini Clerck, 1758, Madrid, 30TVK47, Sanz de Diego, 1885

Xysticus audax (Schranck, 1803), Valdelaguna, 30TVK64, Fernández de Céspedes, 1987

Distribución. Paleártica. Toda la Península ibérica.

Xysticus bliteus (Simon, 1875)*

- Oxyptila blitea

CitAS DE LA CM

Oxyptila blitea Simon, 1875, Valdelaguna, 30TVK64, Fernández de Céspedes, 1987

Oxyptila blitea Simon, 1875, Valdelatas, 30TVK49, Camargo, 1982

DistriBUCIÓN. Mediterránea. Repartida por toda la Península.

Xysticus bufo (Dufour, 1820)

- Thomisus bufo

CiTAS DE LA CM

Oxyptila albimana Simon, 1875, El Escorial, 30TVK09, Fernández Galiano, 1910

Xysticus bufo Dufour, 1820, Montarco, 30TVK57. Depositado en el MNCN 20.02/10017. Revisado por C. Urones (etiqueta antigua como Oxyptila albimana Simon).

Oxyptila albimana Simon, 1875, Pelayos, 30TUK97, Fernández Galiano, 1910. Depositado en el MNCN 20.02/10016. Revisado por C. Urones

Xysticus bufo (Dufour, 1820), La Maliciosa. Srra de Guadarrama, 30TVL11. Material depositado en el MNCN 20.02/10524. Identificado por C. Urones. 
Xysticus bufo (Dufour, 1820), San Agustín de Guadalix, 30TVL40, Ferrández y y Fernández de Céspedes

Proxysticus bufo (Dufour, 1820), Valdelatas, 30TVK49, Camargo, 1982

Distribución. Paleártica occidental. Toda la Península Ibérica.

Xysticus caperatus (Simon, 1875)

- Xysticus caperatus

CitAs DE LA CM

Xysticus caperatus (Simon, 1875), Robledo de Chavela, 30TUK97, Fernández Galiano, 1910

Proxysticus caperatus (Simon, 1875), Valdelatas, 30TVK49, Camargo, 1982

Distribución. Paleártica occidental. Mitad Norte de la Península, falta en Portugal.

Xysticus cor Canestrini, 1873*

- Xysticus cor

CitAs DE LA CM

Xysticus cor Canestrini, 1873, Valdelatas, 30TVK49, Camargo, 1982

Distribución. Sur de Europa. Citada del Algarve, en Portugal y de Gerona y la Sierra de Gredos, en España.

Xysticus cribatus Simon, 1885

- Xysticus cribatus

CitAs DE LA CM

Xysticus cribatus Simon, 1885, Montarco, 30TVK57, Perez de San Román, 1947. Depositado en el MNCN 20.02/10504. Revisado por C. Urones

Xysticus cribatus Simon, 1885, Montejo de la Sierra, 3TTVL54. Material depositado en el MNCN 20.02/10015. Identificado por C. Urones

DisTRIBUCIÓN. Mediterráneo. Solo citada de Granada y de Madrid.

\section{Xysticus ferrugineus Menge, 1876}

- Xysticus ferrugineus

CitAs DE LA CM

Xysticus kochi Thorell, El Escorial, 30TVK09, Fernández Galiano, 1910. Depositado en el MNCN 20.02/10337. Revisado por C. Urones

Xysticus pini Hahn, 1831, San Martín de Valdeiglesias, 30TUK85, Fernández Galiano, 1910. Depositado en el MNCN 20.02/10486. Revisado por C. Urones

Xysticus ferrugineus Menge, 1876, Valdelatas, 30TVK49, Camargo, 1982

DisTRIBUCión. Paleártica occidental. Conocida de toda la Península.

Xysticus grallator Simon, 1932

- Xysticus grallator

CitAs DE LA CM

Xysticus grallator Simon, 1914, Cantoblanco. Alrededores de la UAM, 30TVK49, Urones, Camargo \& Ferrández, 1986

Xysticus grallator Simon, 1914, Madrid, 30TVK48, Urones, Camargo \& Ferrández, 1986
DISTRIBUCIÓN. Mediterránea occidental: España y Córcega. Solo citada de Cáceres y Madrid.

Xysticus kochi Thorell, 1872

- Xysticus Kochii

Citas DE LA CM

Xysticus kochi Thorell, 1872, El Berrueco, 30TVL52, Jiménez, 2002

Xysticus Kochi Thorell, 1872, El Escorial, 30TVK09, Fernández Galiano, 1910. Depositado en el MNCN 20.02/10338. Revisado por C. Urones

Xysticus kochi Thorell, 1872, Valdelatas, 30TVK49, Camargo, 1982

Distribución. Europea. Mitad norte de la Península Ibérica.

Xysticus lanio C.L. Koch, 1835

- Xysticus lanio

Citas de LA CM

Xysticus lanio C.L. Koch, 1835, Aranjuez, 30TVK53, Sanz de Diego, 1885

Xysticus lanio C.L. Koch, 1835, El Berrueco, 30TVL52, Jiménez, 2002

Distribución. Paleártica. Mitad norte de la Península Ibérica.

Xysticus nubilus Simon, 1875*

- Xysticus nubilus

CitAs DE LA CM

Xysticus nubilus Simon, 1875, El Escorial, 30TVK09. Material depositado en el MNCN 20.02/10512. Identificado por C. Urones

Xysticus nubilus Simon, 1875, Madrid, 30TVK47. Material depositado en el MNCN 20.02/10500. Identificado por C. Urones

Xysticus nubilus Simon, 1875, Valdelatas, 30TVK49. Camargo, 1982

DisTRIBUCIÓN. Mediterráneo occidental y también Islas Macaronésicas. Centro de la Península.

\section{Xysticus ovatus Simon, 1876*}

- Xysticus ovatus

CitAs DE LA CM

Xysticus ovatus Simon, 1875, Montarco, 30TVK57. Material depositado en el MNCN 20.02/10508. Identificado por C. Urones

DistriBUCión. Francia y España.

Xysticus sabulosus (Hahn, 1832)

- Thomiusus sabulosus

Citas DE LA CM

Xysticus sabulosus (Hahn, 1832), El Escorial, 30TVK09. Depositado en el MNCN 20.02/10499. Identificado por C. Urones

Xysticus sabulosus (Hahn, 1832), Madrid, 30TVK47, Fernández Galiano, 1910. Depositado en el MNCN 20.02/10490. Revisado por C. Urones 
Xysticus sabulosus (Hahn, 1832), Valdelaguna, 30TVK64, Fernández de Céspedes, 1987

Psammitis sabulosa (Hahn, 1832), Valdelatas, 30TVK49, Camargo, 1982

Distribución. Paleártica. Región central de la Península.

Xysticus semicarinatus Simon, 1932

- Xysticus semicarenatus

Citas DE LA CM

Xysticus semicarenatus Simon, 1932, Peñalara, 30TVL12, Simon, 1932

DistribuCión. Mediterráneo occidental. Mitad Norte y montañas del Sur de la Península Ibérica.

Xysticus ulmi (Hahn, 1832)*

- Thomisus ulmi

CitAs DE LA CM

Xysticus ulmi (Hahn, 1832), Valdelatas, 30TVK49, Camargo, 1982

DisTribución. Paleártica. Solo conocida de Coimbra (Beira Litoral).

\section{Xysticus sp}

CitAs DE LA CM

Xysticus sp, Cercedilla. estación alpina. 30TVL11. Material depositado en el MNCN 20.02710334. Identificado por C. Urones

Xysticus caperatus Simon, 1875, El Escorial, 30TVK09, Fernández Galiano, 1910. Depositado en el MNCN 20.02/10505. Revisado por C. Urones

Xysticus sp, Sierra de Guadarrama. Material depositado en el MNCN 20.02/10510. Identificado por C. Urones.

\section{Familia Titanoecidae Lenthinen, 1967}

Nurscia sequerai (Simon, 1892)*

- Titanoeca sequerai

CitAs DE LA CM

Nurscia sequerai (Simon, 1892), San Agustín de Guadalix, 30TVL40, Ferrández y Fernández de Céspedes

Distribución. Portugal hasta Francia, solo conocida de Portugal.

Titanoeca monticola (Simon, 1870)*

- Dictyna monticola

CitAs DE LA CM

Titanoeca monticola (Simon, 1870), Valdelaguna, 30TVK64 Fernández de Céspedes, 1987

DistribuCión. Mediterráneo occidental. Conocida de Jaén, Gerona, Zaragoza y Beira litoral.

Titanoeca obscura (Walckeaner, 1802)

- Aranea obscura

CITAS DE LA CM

Titanoeca quadriguttata (Hahn, 1833), San Martín de Valdeiglesias, 30TUK85, Fernández Galiano, 1910. Depositado en el MNCN 20.02/10374
DistriBUCiÓN. Paleártica occidental. Citada de las provincias de Navarra, Huesca, Tarragona y Madrid. Falta en Portugal.

Familia Uloboriidae O. Pickard-Cambridge, 1871

Hyptiotes flavidus (Blackwall, 1862)

- Mithras flavidus

CiTAS DE LA CM

Hyptiotes flavidus (Blackwall, 1862), Torrelodones, 30TVK29, Ferrández \& Beamonte, 2005

Distribución. Mediterránea. Conocida de Portugal y de Aragón.

Uloborus walckenaerius Latreille, 1806

- Uloborus walckenaerius

Citas DE LA CM

Uloborus Walckenaerius Latreille, 1806, Pelayos, 30TUK97, Fernández Galiano, 1910. Depositado en el MNCN 20.02/10517

Uloborus Walckenaerius Latreille, 1806, Robledo de Chavela, 30TUK97, Fernández Galiano, 1910. Depositado en el MNCN 20.02/10519

Distribución. Paleártica occidental. Toda la Península ibérica.

Familia Zodariidae Thorell, 1881

Amphiledorus balnearicus Joque \& Bosmans, 2001*

- Amphiledorus balnearicus

CITAS DE LA CM

Amphiledorus balnearicus Joque \& Bosmans, 2001, San Agustín de Guadalix, 30TVL40, Ferrández y Fernández de Céspedes

Distribución. Ibérica. Solo conocida de Andalucia, Murcia, Barcelona y Zaragoza.

Selamia histrionica Simon, 1884*

- Selamia histrionica

Citas DE LA CM

Storena histrionica (Simon, 1884), Valdelaguna, 30TVK64, Fernández de Céspedes, 1987

DisTRIBUCIÓN. Mediterránea occidental. Solo hay una cita previa de Alicante.

Selamia reticulata (Simon, 1870)

- Lachasia reticulata

Citas DE LA CM

Storena reticulata (Simon, 1870), La Acebeda, 30TVL44, Ferrández \& Beamonte, 2005

Storena reticulata (Simon, 1870), El Escorial, 30TVK09, Simon, 1874 
Storena reticulata (Simon, 1870), Robledo de Chavela, 30TUK97, Simon, 1874

Selamia reticulata (Simon, 1870), San Agustín de Guadalix, 30TVL40, Ferrández y Fernández de Céspedes

Storena reticulata (Simon, 1870), Valdelaguna, 30TVK64, Fernández de Céspedes, 1987

Storena reticulata (Simon, 1870), Valdelatas, 30TVK49, Camargo, 1982

DisTRIBUCIÓN. Mediterráneo occidental. Toda la Península ibérica.

Zodarion affine (Simon, 1870)

- Enyo affine

CITAS DE LA CM

Zodarion affine (Simon, 1870), El Escorial, 30TVK09, Fernández Galiano, 1910

Distribución. España. Citado solo de Alicante, Cádiz y Madrid.

Zodarion alacre (Simon, 1870)

- Enyo alacris

CiTAS DE LA CM

Zodarion alacre (Simon, 1870), Aranjuez, 30TVK53, Denis, 1937 Zodarion alacre (Simon, 1870), El Berrueco, 30TVL52, Jiménez, 2002

DistriBución. Ibérica. Toda la Península Ibérica.

Zodarion fulvonigrun (Simon, 1874)*

- Enyo fulvo-nigra

CITAS DE LA CM

Zodarion fulvonigrum (Simon, 1874), Valdelatas, 30TVK49, Camargo, 1982

DisTRIBUCIÓN. Mediterráneo occidental. Citada tan solo de Salamanca.

Zodarion fuscun (Simon, 1870)

- Enyo fusca

CitAs DE LA CM

Enyo fusca Simon, 1870, El Escorial, 30TVK09, Simon, 1870

Zodarion fuscum (Simon, 1870), Robledo de Chavela 30TUK97, Fernández Galiano, 1910

DisTRIBUCIÓN. Mediterránea occidental. Toda la Península Ibérica.

Zodarion styliferum (Simon, 1870)

- Enyo stylifera

CitAs DE LA CM

Zodarion styliferum (Simon, 1870), El Pardo, 30TVK38, Bosmans, 1994

Zodarion styliferum (Simon, 1870), Madrid. Casa de Campo, 30TVK47, Bosmans, 1994

Zodarion styliferum (Simon, 1870), San Agustín de Guadalix, 30TVL40, Ferrández y Fernández de Céspedes

Zodarion styliferum (Simon, 1870), Sierra de Guadarrama, Simon, 1870

Zodarion styliferum (Simon, 1870), Torrejón de Ardoz, 30TVK57, Bosmans, 1994. Depositado en el AMNH
Zodariun styliferum (Simon, 1870), Valdelaguna, 30TVK64, Fernández de Céspedes, 1987

Zodarium marginiceps Simon, 1914, Valdelatas, 30TVK49, Camargo, 1984. Incorrectamente identificado.

Distribución. Península Ibérica y Madeira. Toda la Península Ibérica.

\section{AGRADECIMIENTOS}

Queremos expresar aquí nuestro agradecimiento a Miguel Villena -conservador de invertebrados del Museo Nacional de Ciencias Naturales- que nos facilitó la información sobre las arañas de la Comunidad de Madrid existentes en las colecciones a su cargo y también a José Beamonte que colabora con el Museo en las tareas de informatizar las citadas colecciones. Este trabajo ha sido subvencionado por el proyecto GR/AMB/0750/2004 de la Comunidad de Madrid.

\section{Referencias}

BACELAR, A., 1932. Inventario das Aranhas migalomorfas da Península Ibérica. Asociación Española para el progreso de las Ciencias: 17-24.

BARrientos, J. A., 1980. L'identité de Tegenaria feminea E. Simon, 1870 (Agelenidae, Araneae). Veme Colloque Arachnologique, Barcelona (1979): 15-20.

BARrientos, J. A., 1984. Status taxonomiques des Oxyopes Latreille, de la Peninsule Iberique (Araenae: Oxyopidae). Revue Arachnologique, 8(4): 153-159.

Barrientos, J. A., BACH, C \& GaJu, M., 1986. Sobre algunas arañas de la cuenca del Bembezar (Cordoba). El género Arctosa. Miscel-lània Zoològica, 9: 163-169.

BARrientos, J. A. \& RiberA, C., 1988. Algunas reflexiones sobre las especies del grupo " atrica " en la Península Ibérica (Araneae: Tegenaria). Revue Arachnologique, 7(4): 141-162.

Blasco, A., 1986. Artrópodos epigeos del macizo de San Juan de la Peña (Jaca: provincia de Huesca): Arañas Migalomorfas. Pirineos, 126(2): 5-28.

Bolívar, I., 1875. Enumeración de algunos Aracnidos de España. Actas de la Sociedad española de Historia Natural, 4: 37.

Bosmans, R., 1994. Revision of the genus Zodarion Walckenear, 1833 in the Iberian Peninsula and Balearic islands (Araneidae; Zodariidae). Eos, 69: 115-142.

Bosmans, R. \& VAn DE KeER, R., 1985. Catalogue des araignées des Pyrénées. Espèces citées, nouvelles recoltes, bibliographie. Institut Royal des Sciences Naturelles de Belgique, Documents de Travail, 23: 168: 1985

BRAUM, R., 1965. Beitrag zu einer revison der paläarktischen Arten der Philodromus aureolus Gruppe (Arach., Araneae). I. Morphologisch-systematischer Teil. Senckbergiana biologica, 46(5): 369-428. 
Brignoli, P. M., 1971. Su alcune Tegenaria d'Ispagna (Araneae, Agelenidae). Memorie del Museo Civico di Storia Naturale di Verona, [1970], 18: 307-312.

Brignoli, P. M., 1976. Beitrage zur Kenntnis der Scytodiidae (Araneae). Revue Suisse de Zoologie, 83(1): 125-191.

Brignoli, P. M., 1978. Quelques notes sur les Agelenidae, Hahnidae, Oxyopidae et Pisauridae de France et d'Espagne (Araneae). Revue Suisse de Zoologie, 85(2): 265-294.

Camargo, M., 1982. Estudio de los Araneidos (Araneae) de Valdelatas. Tesis de Licenciatura. Universidad Autónoma de Madrid. 295 pp. Inédita.

CAmargo, M. \& Ferrández, M. A., 1985. Redescripción y afinidades de Castianeira badia Simon,1877 (Araneidae: Clubionidae). Miscel-lània Zoològica, [1984], 8: 297-300.

CARDoso, P., 2000. Portuguese spiders (Araneae) a prelimnary checklist. Ekólogia (Bratislava), 19(Supplement 3): 19-29.

CAstro, A. DE, 2005. Catálogo preliminar de las arañas del País Vasco. Munibe, Suplemento 21: 45-68.

Dalmas, R. DE, 1916. Revison du genre Orchestina E. Simon (Arach. Oonopidae) suivie de la description de nouvelles espèces du genre Oonops et d'une étude sur les Dictinidae du genre Scotolathys. Annales de la Société entomologie de France, 85: 203-258.

DENIS, J., 1937. Contribution à l'etude des araignées du genre Zodarion Wackenaer. Festschrift für Prof. Dr. Embrik Strand, 3: 1-50, pl. I-VIII.

DENIS, J., 1962. Quelques araignées d'Espagne centrale et septentrionale et remarques synonimiques. Bulletin de la Société d'Histoire Naturelle de Toulouse, 97(12): 276-292.

Dufour, L., 1861. Sur 1'Epeira serice et le Pompilus croceicornis avec quelques considerations sur leur habitat geographique. Annales de la Société Entomologique de France, (4), 1: 5-7.

FERnÁNDEZ Bourgon, C., 2003. Las arañas urbanas. La Tarantúla, 2: 74-81

FERnÁNDEZ DE CÉsPedes, H., 1987. Contribución al estudio de la fauna de arañas (Arachnida: Araneae) de los eriales de la provincia de Madrid. Tesis de licenciatura. Universidad Complutense de Madrid. 181 pp. Inédita.

Fernández Galiano, E., 1910. Datos para el conocimiento de la distribución geografica de los Aracnidos de España. Memorias de la Real Sociedad española de Historia Natural, 6: 1-86.

FERrÁnDEZ, M. A., 1984. Nota sobre los Dysderidae Ibericos III. Una nueva especie de Dysdera de la provincia de Madrid. Eos, [1983], 59: 41-44.

FERrÁNDEZ, M. A., 1985. Notes on the Iberian Dysderidae. Three New Species belonging to the genus Dysdera Latreille, 1804 (Araneae). Miscel-lània Zoològica, [1984], 8: 189-195.
FERRÁnDEZ, M. A., 1986. Las especies ibéricas del género Harpactocrates Simon, 1914 (Araneida; Dysderidae). Actas del X Congreso Internacional de Aracnología, Jaca, 1: 337-348.

Ferrández, M. A., 1987. Los Dysderidae (Arachnida: Araneae) de la Peninsula Ibérica. Tesis Doctotal. Universidad Complutense de Madrid. 488 pp. Inédita.

FerrándeZ, M. A. \& Alberdi, J. M., 2005. Nuevas de Mimétidos ibéricos (Araneae: Mimetidae). La Tarantúla, 3: 9-25.

Ferrández, M. A. \& BeAmonte, J., 2005. Nuevas citas para la Península Ibérica 1/2005. La Tarantúla, 3: 67-71.

Ferrández, M. A. \& Fernández de Céspedes, H., 1990. Nuevos datos sobre las especies ibéricas del género Harpactea Bristowe, 1939 (Araneae: Dysderidae) Boletín de la Real Sociedad española de Historia Natural (Sección Biológica), 86(1-4): 39-53.

Frade, F. \& Bacelar, A., 1931. Revision des Nemesia de la faune iberique et descriptions d'especees nouvelles de ce genre. Bulletin du Muséum Nationale d'Histoire Naturelle, 2(3)2: 222-238.

Grimm, U., 1986. Die Gnaphosidae Mitteleuropas (Arachnida, Araneae). Abhandlungen des Naturwissenschaftlichen Vereins in Hamburg, N.F., 26: 1-318.

JimÉnEZ-VAlverde, A., 2002. Presencia en la Comunidad de Madrid (España central) del endemismo ibérico Ozyptila umbraculorum Simon, 1932 (Araneae, Thomisidae). Revista Ibérica de Aracnología, 6: 225-227.

JimÉnEZ-VAlVerde, A., BARriga Bernal, J. y Morano, K., 2004. Datos interesantes sobre la distribución de Araniella opistographa (Kulczynski, 1906) y Araniella incospicua (Simon, 1874) (Aranae: Araneidae) en la Península Ibérica. Revista Ibérica de Aracnología, 9: 269-270.

JimÉnEZ-VAlVERde, A. \& LÓPEZ-Colón, J. I., 2003. Primera cita para Madrid de Menemerus taeniatus (L. Koch, 1867) (Araneae: Salticidae) para España. Revista Ibérica de Aracnología, 7: 175-176.

JimÉneZ-VAlVerde，A. \& WesolowsKA, W., 2005. Synageles albotrimaculatus (Lucas, 1845) (Araneae, Salticidae) a spider new to Spain. Bulletin of the British Arachnological Society, 13(4): 122-124.

Koch, L., 1868. Die Arachnidengattungen Amaurobius, Coelotes und Cybaeus. Abhanlungen der naturhistorichen Gessellschaft - Nürnberg, 1868: 1-52.

Luna, J. \& Delgado, R., 2002. Cata aracologica: Los Licósidos (Araneae, Lycosidae) de la Dehesa Bonita. La Tarantúla, 1: 7-14.

Machado, A. De Barros, 1942. A coleçao de Aranhas cavernicolas do Museo Nacional de Ciencias Naturais de Madrid. Anales Asociación española para el Progreso de las Ciencias, 7: 806-820.

Melic, A., 2000. Arañas de Aragón (Arachnida: Araneae). Catalogus de la Entomofauna aragonesa, 22: 3-40. 
Morano, E., 1984. Contribución al conocimiento de las familias Araneidae y Tetragnathidae (Arachnida: Araneae) de la Península Ibérica. Tesis de Licenciatura. Universidad Complutense de Madrid. 548 pp. Inédita.

Morano, E., 2001. Especies nuevas o poco conocidas de arañas (Arachnida, Araneae) de la fauna ibérica. Revista Ibérica de Aracnología, 3: 67-68.

Morano, E., 2002. Las especies de Larinoides Caporiacco, 1934 (Araneae, Araneidae) de la Península Ibérica. Revista Ibérica de Aracnología, 5: 65-74.

Morano, E., 2005a. Introduccion a la diversidad de las arañas iberbaleares. Munibe, Suplemento 21: 93-137.

Morano, E., 2005b. Catálogo de las Arañas de la Península Ibérica. http://aracnologia.enor.org/cata intro es.htlm.

Morano, E. \& Ferrández, M. A., 1985a. Araneus alpicus (C.L. Koch, 1869) nueva especie de la familia Argiopidae (Araneidae) para la Península Ibérica. Boletín de la Asociación española de Entomología, 9: 31-34.

Morano, E. \& Ferrández, M. A., 1985b. Especies nuevas o de interés de la familia Araneidae Latreille, 1806 (Arachnida; Araneae) de la fauna ibérica. Miscel-lània Zoològica, [1985], 9 : 171-178.

Morano, E. \& Ferrández, M. A., 1986. Contribución al conocimiento de la familia Araneidae Latreille, 1806 de la provincia de Madrid. Graellsia, 42: 161-174.

Ortuño, V., 1992. Los Coleópteros carábidos. Morfología, biología y sistemática. Fauna de la Comunidad de Madrid. Ministerio de Medio Ambiente, Organismo Autonomo de Parques Nacionales, Serie Técnica. Madrid. 269 pp.

OrtuÑo, V., 2002. Estado de conocimiento de los artrópodos de España. In: F. D. Pineda, J de Miguel, M. A. Casado y J. Montalvo (coords.-eds.). La Diversidad Biologica de España. Pearson Educación. Madrid: 209-234.

Pérez, J. A., 1986. Los Gnaphosidae (Araneae) de la colección del Museo Nacional de Ciencias Naturales (MNCN) de Madrid. Actas X Congreso Internacional de Aracnología, Jaca, 1: 420-421.

PÉREZ ARCAS, L., 1872. Noticia sobre la Epeira sericea Ol. Anales de la Sociedad española de Historia Natural, 1(Actas): 25.

Pérez de SAn Román, F. \& Ruiz de ZÁrate, F., 1947. Catálogo de las especies del Orden Araneae citadas en España despues de 1910. Boletín de la Real Sociedad española de Historia Natural, 45: 417-491.

PICKARD-CAMBRIDGE, O., 1875. On some new species of Erigone. part I. Proceedings of the zoological Society of London, 1875: 190-224. Pl. XXVII-XXIX.

PLATNICK, N. I., 2005. The world spiders catalog versión 5.0. http://research.amnh.org/entomology/spiders/ catalog./index.htlm.
Platnick, N.I. \& MurPhy, J. A., 1984. A Revision of the Spider genus Trachyzelotes and Urozelotes (Araneae: Gnaphosidae). American Museum Novitates, 2792: 130.

Platnick, N.I. \& Murphy, J. A., 1996. A review of the zelotine ground spiders genus Setaphis (Araneae: Gnaphosidae). American Museum Novitates, 3162: $1-23$.

RAmos, $M^{\mathrm{a}}$. A., 2002. Riqueza faunística de la península ibérica e Islas Baleares. El proyecto fauna ibérica. In: F. D. Pineda, J. de Miguel, M. A. Casado y J. Montalvo (coords.-eds.). La Diversidad Biologica de España. Pearson Educación. Madrid: 197-208.

Ribera, C., Ferrández, M. A. \& Pérez, J. A., 1987. Los Hersilidos (Araneae: Hersiliidae) de la fauna ibérica. Miscel-lània Zoològica, [1986], 10: 97-103.

SÁnchez, I., 2003. Catálogo preliminar de los Araneae de la provincia de Cádiz. Revista de la Sociedad Gaditana de Historia Natural, 3: 199-216.

SANZ DE Diego, M., 1885. Lista de Arácnidos recogidos por... Actas de la Sociedad española de Historia Natural, 14: 38-41.

SCHENCKEL, E., 1938. Spinnentiere von der Iberische Halbinsel gesammelt von Prof. Dr. O.Lumblad JuliAugust 1935. Arkiv für Zoologie, 30A(24): 1-29.

Simon, E., 1868. Monographie des espèces europeennes de la famille des Attides (Attidae SundevallSaltigrade Latreille). Annales de la Société entomologique de France, (4), 8: 11-72, 529-726, pl. V-VII.

SimON, E., 1870a. Araignees nouveaux ou peu connus du midi de 1'Europe (1er memoire). Memoire de la Société royal des sciencies de Liège: 1-90.

Simon, E., 1870b. Sur les Araneides de la famille des Enydes qui habiten 1'Espagne et le Maroc. Revue et Magazine de Zoologie, (2), 22: 51-54, 97-103 y 142148.

SimON, E., 1873. Note sur les espèces européennes de la famille Eresidae. Annales de la Société entomologique de France, (5), 3: 335-358.

Simon, E., 1874. Les Arachnides de France. Tome I. Librairie Encyclopédique de Roret. Paris. 272 pp.

Simon, E., 1875. Les Arachnides de France. Tome II. Librairie Encyclopédique de Roret. Paris. 350 pp.

Simon, E., 1877. Description de deux espèces nouvelles d`Arachnides du genre Agroeca. Annales de la Société entomologique de France, (5), 7: CLXXXIX

Simon, E., 1878. Les Arachnides de France. Tome IV. Librairie Encyclopédique de Roret. Paris. 334 pp.

SimON, E., 1881. Descriptions d'arachnides nouveaux d'Espagne et de Portugal. Anales de la Sociedad española de Historia Natural, 10: 133-136.

Simon, E., 1914. Les Arachnides de France. Tome VI. $1^{\text {ème }}$ partie. Librairie Encyclopédique de Roret et L. Mulo. Paris. i-vi, 308 pp. 
Simon, E., 1932. Les Arachnides de France. Tome VI. $4^{\text {ème }}$ partie. Librairie Encyclopédique de Roret et L. Mulo. Paris. pp. 773-978.

Simon, E., 1937. Les Arachnides de France. Tome VI. $5^{\mathrm{èm}}$ et dernier partie. Librairie Encyclopédique de Roret et L. Mulo. Paris. pp. 979-1298.

Tekfer, G., Bosmans, R., Melic, A. \& Rego, F., 2003. The spiders of Portugal: some additions to the current checklist (Araneae). Revista Ibérica de Aracnología, 7: 251-255.

Urones, C., 1989. Nuevos datos de Clubionidae (Araneae) Ibéricos. Miscel-lània Zoològica, 13: 55-61.

Urones, C., 1995. Catálogo y atlas de las arañas Philodromidae Ibéricas. Graellsia, 51: 55-81.

Urones, C., 1996. Catálogo y Atlas de las arañas de la familia Anyphaenidae Bertkau, 1878 de la Península Ibérica e Islas Baleares. Graellsia, 52: 73-80.
Urones, C., 2004. El género Micrommata (Araneae, Sparassidae) en la Península Ibérica. Revista Ibérica de Aracnología, 10: 41-52.

Urones, C., Camargo, M. \& Ferrández, M. A., 1983. Presencia en la Península Ibérica de Xysticus grallator Simon, 1914 (Araneida: Thomisidae) con comentarios acerca de las especies pertenecientes al grupo $2^{\circ}$ de Simon 1914. Actas del $1^{\circ}$ Congreso Ibérico de Entomología. León, 2: 781-784.

WesolowsKa, W., 1986. A Revision of the genus Heliophanus C.L. Koch, 1833 (Aranei: Salticidae). Annales Zoologici, 40(1): 1-254.

Wunderlich, J., 1994. Beschreibung der neuen Gattung Phrurolinillus der familie Corinnidae aus Europa (Arachnida; Araneae). Beiträge zur Araneologie, 4: 739-742. 